\title{
Distortional eigenmodes and homogeneous solutions for semi-discretized thin-walled
} beams

\author{
Jönsson, Jeppe; Andreassen, Michael Joachim
}

Published in:

Thin-Walled Structures

Link to article, DOI:

10.1016/j.tws.2010.12.009

Publication date:

2011

Document Version

Early version, also known as pre-print

Link back to DTU Orbit

Citation (APA):

Jönsson, J., \& Andreassen, M. J. (2011). Distortional eigenmodes and homogeneous solutions for semi-

discretized thin-walled beams. Thin-Walled Structures, 49(6), 691-707. https://doi.org/10.1016/j.tws.2010.12.009

\section{General rights}

Copyright and moral rights for the publications made accessible in the public portal are retained by the authors and/or other copyright owners and it is a condition of accessing publications that users recognise and abide by the legal requirements associated with these rights.

- Users may download and print one copy of any publication from the public portal for the purpose of private study or research.

- You may not further distribute the material or use it for any profit-making activity or commercial gain

- You may freely distribute the URL identifying the publication in the public portal

If you believe that this document breaches copyright please contact us providing details, and we will remove access to the work immediately and investigate your claim. 


\title{
Distortional eigenmodes and homogeneous solutions for semi-discretized thin-walled beams
}

\author{
J. Jönsson, M. J. Andreassen \\ Technical University of Denmark, Department of Civil Engineering, Brovej Building 118, \\ DK-2800 Kgs. Lyngby
}

\begin{abstract}
The classical Vlasov theory for torsional analysis of thin-walled beams with open and closed cross-sections can be generalized by including distortional displacement fields. We show that the determination of adequate distortional displacement fields for generalized beam theory (GBT) can be found as part of a semi-discretization process. In this process the cross-section is discretized into finite cross-section elements and the axial variation of the displacement functions are solutions to the established coupled fourth order differential equations of GBT. We use a novel finiteelement-based displacement approach in combination with a weak formulation of the shear constraints and constrained wall widths. The weak formulation of the shear constraints enables analysis of both open and closed cell crosssections by allowing constant shear flow. We use variational analysis to establish and clearly identify the homogeneous differential equations, the eigenmodes, and the related homogeneous solutions. The distortional equations are solved by reduction of order and solution of the related eigenvalue problem of double size as in non-proportionally damped structural dynamic analysis. The full homogeneous solution is given as well as transformations between different degree of freedom spaces. This new approach is a considerable theoretical improvement, since the obtained GBT equations found by discretization of the cross-section are now solved analytically and the formulation is valid without special attention also for closed single or multi cell cross-sections. Further more the found eigenvalues have clear mechanical meaning, since they represent the attenuation of the distortional eigenmodes and may be used in the automatic meshing of approximate distortional beam elements. The magnitude of the eigenvalues thus also gives the natural ordering of the modes.
\end{abstract}

Key words: Distortion, Warping, Generalized beam theory, Thin-walled beams, Beam theory, Semi-discretization.

\section{Introduction}

Thin-walled structural elements are extremely efficient due to the minimization of the thickness-to-width ratio of the cross-section walls, and the thin walls are a primary aspect in the behavior and design. The increasing slenderness leads to the insufficiency of both ordinary Euler-Bernoulli beam theory with St. Venant torsion and Vlasov beam theory for thin-walled beams, [1]. An important feature missing in these theories is the distortion of the cross-sections, which do not maintain their shape, but distort and buckle. Several formulations including transverse distortional displacements have been proposed for analysis of both open and closed

Email addresses: jej@byg.dtu.dk (J. Jönsson), mican@byg.dtu.dk (M. J. Andreassen)

Preprint submitted to Thin-Walled Structures cross-sections. One formulation which has been very successful is the generalization of classic Vlasov beam theory for open cross-sections to include distortion. It has been based on the kinematic assumption of negligible shear strain along the centre lines of the crosssection walls. This formulation is known as GBT (generalized beam theory) and it was initially proposed by Schardt [2] in 1966 as a generalization of the theory of bending (Verallgemeinerte Technisch Biegetheorie, VTB in German). For closed (single or multi celled hollow) thin-walled cross-sections, Schardt shows in his presentation of GBT in [3], that the theory needs a relaxation of the Vlasov assumption of negligible shear strain in order to include the warping deformation associated with the "Bredt's shear flow" around each cell. However it complicates the solution of the GBT equations by introducing non negligible shear coupling terms (off

December 16, 2010 
diagonal) in the GBT equations as can be seen in recent GBT formulations for closed thin-walled cross-sections, e.g. [4], [5] and [6]. In this paper we will therefore adhere to the definition of the warping function given by Kollbrunner \& Hajdin, [7], which adds the integral of the shear flow strains, see also [8] and [9]. Since GBT is developed as a generalization of beam bending it leads to orthogonal warping modes. Thus the final GBT equations are decoupled with respect to normal stresses and transverse stresses, however the shear coupling terms are neglected or in case of closed cells given special attention. The basic cross-section deformation modes of GBT are obtained by separately identifying conventional beam deformation modes and solving the eigenvalue problem defined by the warping stiffness matrix and the transverse deformation stiffness matrix. When solving the GBT equations the shear coupling stiffness terms are neglected. This corresponds to modal analysis with orthogonal (Rayleigh) damping in dynamic structural analysis. The solution of the shear coupled GBT equations for closed cross-sections was published by Hanf only in his thesis, [10]. GBT was generally not so known in the international research community until Davies, see [11], presented first-order GBT analysis. A distortional theory which generalizes Vlasov beam theory by including the modified definition of the warping function and one distortional mode was presented by Jönsson [12]. In that work the analytical solution of coupled torsional and distortional equations were found by reduction of order and solution of the related eigenvalue problem as in the present work. GBT is devoted to (first order) distortional displacement analysis including the identification of distortional modes, as well as (second order) linear buckling of thin-walled members, and it has fostered a lot of research. Silvestre $\&$ Camotim extended the theory to include orhotropic materials, see [13] and [14]. Experimental verifications have also been presented, see for example Rendek \& Balaz [15]. Silvestre [16] presents buckling solutions as well as non-linear post buckling solutions. However in order to perform post buckling analysis with GBT, which is essentially a beam theory and not a plate theory, Silvestre finds it is necessary to include additional transverse extension modes and shear modes, as well as modified constitutive relations. In the following we will refer to these kinds of modes as other modes and the related method as extended GBT. For an overview and information about the research and development of GBT see Camotim et al. [17] and [18].

The innovative theoretical developments performed in this paper by introducing semi-discretization lead towards a modified formulation of GBT, in which the ro- tational degrees of freedom are included, thus including local plate modes in the formulation even for the simplest discretization. The elimination of these rotations could perhaps be advantageous if one wants to perform a modal decomposition of buckling displacements into distortional buckling mode and local plate buckling mode, however we then rely on a coarse discretization. The methods developed by Schardt, Silvestre, Camotim and co-workers will therefore be applicable for the presented modified formulation. The presented modified GBT formulation for thin walled beams with both open and closed (single or multi cell) cross-sections can be regarded as an extension of classical Vlasov thin-walled beam theory to include distortional deformation modes as well as constant shear flows in the walls of the cross-section, see [1], [7] and [19]. It makes it possible to analyze thin-walled members with cross-section distortion and local plate behavior in a one-dimensional formulation through the linear combination of pre-established modes of deformation. However in this paper we find the analytical homogeneous solution to the differential GBT equations (obtained by semi-discretisation), using methods similar to Hanf [10] and Jönsson [12], this also (through the magnitude of the eigenvalues) gives a much better knowledge of the length scales of the modes. Alternatively the GBT equations may just as well have been solved using the approximate engineering methods (in which the shear coupling terms are neglected) producing orthogonal axial and transverse normal stress modes. In conventional GBT these modes are used as shape functions in a virtual work or potential energy formulation leading to finite GBT beam elements. However the discretization has to be performed without prior knowledge of the problem length scales of the individual modes. Buckling analysis using GBT beam elements is an alternative to the use of finite-strip methods (FSM), see [20]. However GBT is as its name states essentially a beam theory, whereas FSM essentially is based on plate theory. Therefore FSM does not contain a natural decomposition into basic beam, distortional, local and other modes. Further more conventional GBT does not contain other modes as mentioned above. Since the modal decomposition may lead to advantages in design of thin-walled structures using FSM a great deal of work has been performed by Ádány \& Schafer to develop a constrained finite strip method (cFSM) and modal decomposition methods for open (single-branched) crosssections, see [21], [22] and [23]. The modal approaches of extended GBT and cFSM formulations have recently been compared in [24]. The formulations in this paper only consider the (first order) homogeneous linear 
displacements of GBT, since the main goal has been to identify a theoretically sound formulation of the end effects or in other words find the eigensolutions for the full displacement field including the variation in the axial member direction, see the treatment of end effects by Timoshenko [25].

Let us briefly describe the contents of the following sections and illuminate the process. In the theories of beams, the displacements assumed are typically separated into a sum of (orthogonal) displacement fields. In the following sections, only one of these displacement fields is considered in the variational formulation. The basic kinematic assumptions of these displacement fields are introduced in Section 2. The displacements are separated into the product of cross-section displacement functions and the axial variation functions. The strain fields are derived, and a weak formulation of the shear constraints is described for later use. Simple constitutive energy assumptions in Section 3 lead to the formulation of the elastic energy potential. In Section 4 , the cross-section is discretized by straight finite elements in which the local transverse displacements and the warping displacements are interpolated. The element interpolation functions are introduced and the elastic potential energy is formulated in a semidiscretized form. At this stage, the interpolated displacements have not yet been constrained by assumptions about shear and constant width of wall elements. Section 5 is split into three main steps leading to the final distortional differential equations in which all conventional beam modes have been eliminated and all constraints introduced. In Step I, the weak form of the shear constraint equations are introduced, and by taking adequate variations in the potential energy, the pure axial extension mode and its homogeneous solution is identified and eliminated. In Step II, the constraint equations relating to the assumption of a constant wall width are introduced, and the rigid translations and the rotational cross-section displacement eigenmodes are identified and orthogonalized. The constrained degrees of freedom and the two degrees of freedom related to the rigid translations are eliminated, leading to a condensed set of coupled fourth order differential equations. In Step III, the order of the differential equations is reduced by doubling the number of equations through the introduction of a state vector with components of different differentiation levels. The pure St. Venant torsional mode is identified as an eigenmode with its linear axial solution, St. Venant torsion is eliminated, and the final coupled differential equations are revealed. In Section 6, the distortional eigenvalue problem is solved and the solution functions ordered in a matrix format.
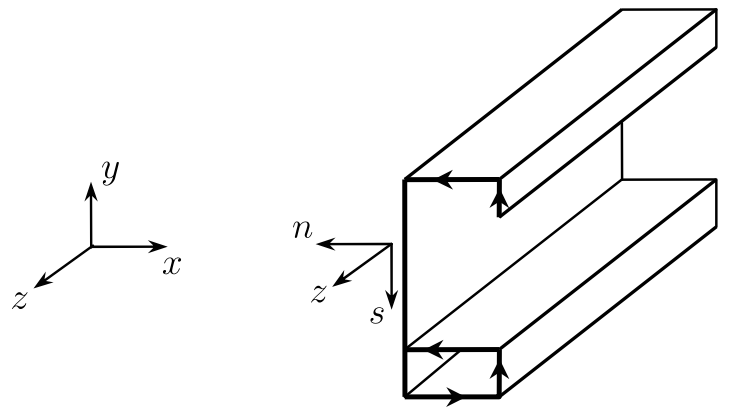

Figure 1: Global and local Cartesian reference frames.

In section 7, the eigenmodes and solutions are assembled in modal vectors and the back substitution steps for the eliminated degrees of freedom are recapitulated in a matrix format. The full homogeneous solution of the GBT differential equations is formulated in Section 8 and the handling of complex eigenvectors and eigenvalues is described. Examples are given in Section 9, and in Section 10, the degree-of-freedom spaces of the finite-element formulation and the GBT formulation are treated and transformations between these are given. Finally Section 11 is devoted to the determination of solution constants using displacement boundary conditions which are relevant for the finite-element formulation of an analytical GBT beam element.

The present paper does not take distributed loads into account, but they can be incorporated in the formulation leading to the addition of inhomogeneous solution functions. In a following companion paper we will address the solution of the inhomogeneous GBT equations, since we in the development stage of the present formulation know that the eigenmodes are needed in order to decouple the reduced order non-homogeneous differential equations as well as the conventional beam equations.

\section{Basic kinematic assumptions}

The prismatic thin-walled beam is described in a global Cartesian $(x, y, z)$ coordinate system where the $z$-axis is in the longitudinal direction of the beam, see Figure 1. A cross-section coordinate $s$ is introduced as a curve parameter which runs through the section along the center line and $n$ is the coordinate along the local normal. Subscripts $n$ and $s$ are used for the components in the local coordinate system corresponding to the normal and tangential directions. Subscripts following a comma are used for derivatives, for example $u_{n, s s}=d^{2} u_{n}(s) / d s^{2}$ or $u_{s, n}=\partial u_{s}(s, n) / \partial n$. A prime, ${ }^{\prime}$, is used for the axial derivative, $d / d z$. 


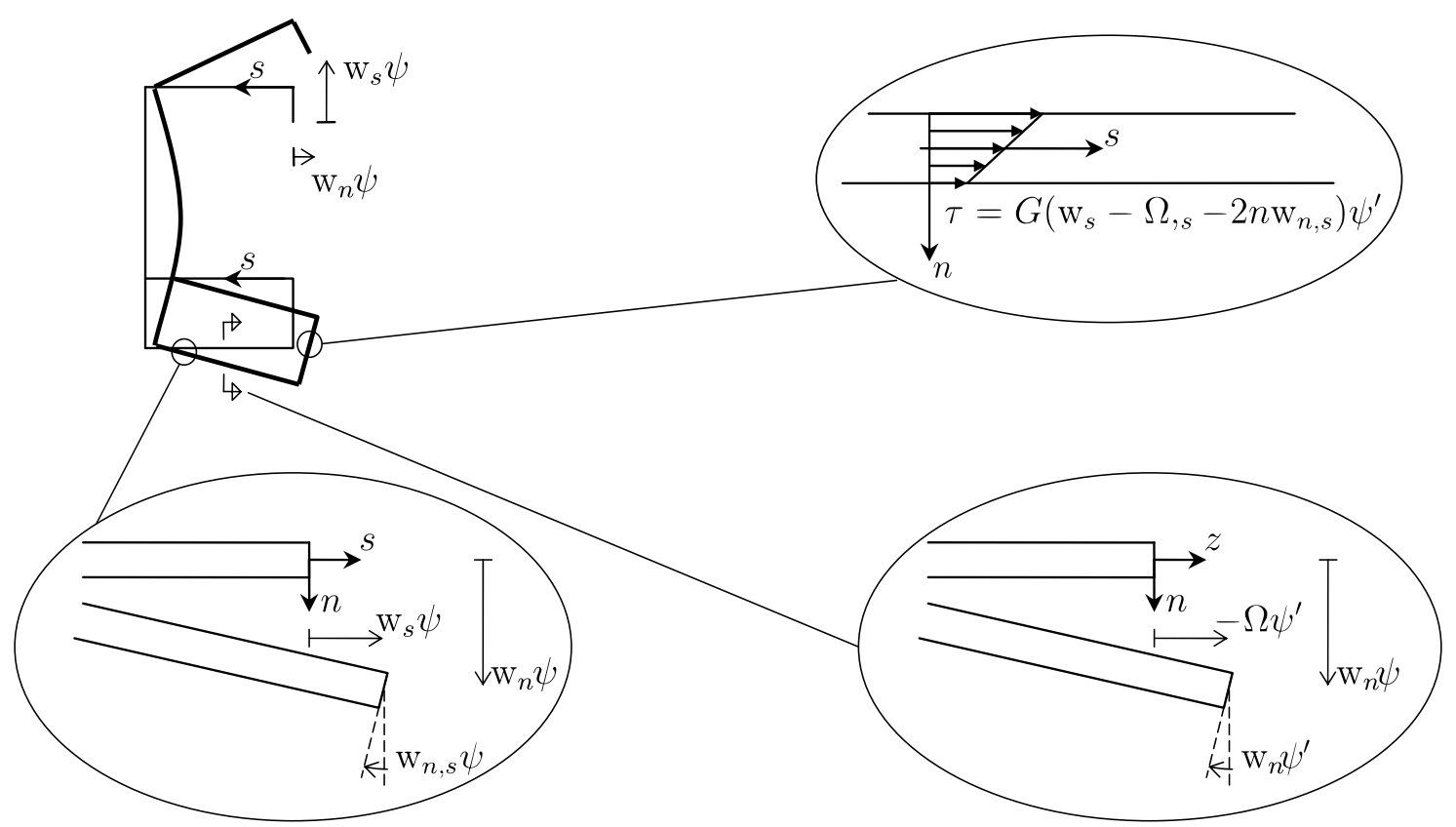

Figure 2: Local components of displacements and assumed shear stresses.

The theories of beams are derived on the basis of assumed displacement fields which correspond to extension, flexure, torsion, warping and distortional displacements. This corresponds to a modal separation in which each mode has a set of transverse and axial displacement fields that may be coupled. Each of these crosssection displacement fields is factorized in a displacement mode which is a function of the in-plane coordinates, multiplied by a function of the axial coordinate, which describes the axial variation of the mode. In the following, we propose a method for finding these displacement modes, including global, distortional and local modes, as the eigenmodes of the corresponding homogeneous set of equilibrium equations and axial variation functions corresponding to the eigenvalues.

In the definition of the displacements and strains, the influence of curved cross-section walls is neglected and it is assumed that the radius of curvature is sufficiently large, so that curvature effects vanish. The local effects at corners and joints are also neglected. Only shear contributions from torsion and shear flow around cells will be allowed. For one displacement mode, the components $u_{n}$ and $u_{s}$ of the in-plane cross-section displacements in the local coordinate system at a point $(n, s)$ in the cross-section, are introduced as

$$
\begin{aligned}
& u_{n}(s, z)=\mathrm{w}_{n} \psi \\
& u_{s}(n, s, z)=\left(\mathrm{w}_{s}-n \mathrm{w}_{n, s}\right) \psi
\end{aligned}
$$

Here $\mathrm{w}_{s}(s)$ and $\mathrm{w}_{n}(s)$ are the local displacements of the centerline as shown in Figure 2, and $\psi(z)$ is the function which describes the axial variation of the inplane distortional displacements. The axial displacements $u_{z}(n, s, z)$ generated by the in-plane distortional displacements are introduced as

$u_{z}(n, s, z)=-\left(\Omega+n \mathrm{w}_{n}\right) \psi^{\prime}$

Here the axial (warping) displacement mode $\Omega(s)$ has been included with a variation corresponding to the negative axial derivative of the axial variation factor, $-\psi^{\prime}$, and due consideration of local transverse variation through the term $n \mathrm{w}_{n}$. Thus neglecting shear deformation contributions which are not related to St. Venant torsion and torsional shear flow around closed cells.

It is convenient at this stage to note that pure axial extension (where $\Omega=1$ and $\mathrm{w}_{s}=\mathrm{w}_{n}=\mathrm{w}_{n, s}=0$ ) is embedded in this formulation. However, since pure extension in the present formulation, does not involve transverse displacements, the axial variation $-\psi^{\prime}(z)$ need not be taken as the derivative of a function, but just a function which we will be introducing as $\zeta(z)=-\psi^{\prime}(z)$ at a later stage.

The axial strains corresponding to the given displacements are

$\varepsilon=u_{z}^{\prime}=-\left(\Omega+n \mathrm{w}_{n}\right) \psi^{\prime \prime}$

The cross-section distortional strains are 
The engineering shear strain in the walls of the crosssection becomes

$$
\gamma=\gamma_{z s}=u_{z, s}+u_{s, z}=\left(\mathrm{w}_{s}-\Omega,,_{s}-2 n \mathrm{w}_{n, s}\right) \psi^{\prime}
$$

To cope with the shear flow around closed cells, we introduce the shear strain in the middle of the wall as

$\bar{\gamma}_{d} \psi^{\prime}=\left(\mathrm{w}_{s}-\Omega,{ }_{s}\right) \psi^{\prime}$

Bernoulli beam theory is based on the assumption of negligible shear strain and sets the shear strain equal to zero and thus determines the warping displacements (flexural modes) by the differential equation $\Omega,{ }_{s}=\mathrm{w}_{s}$. This means that the formulation of Bernoulli beam theory does not include shear contributions and the axial equilibrium equation of a section cut-out is not fulfilled, which leads to the use of Grashof's method for the determination of the shear stresses. However, if we are to analyze closed cross-sections as in Vlasov beam theory, see [7], we have to allow for a constant shear flow around the cells and the warping of the cross-section then has to be determined by the differential equation $\Omega,{ }_{s}=\mathrm{w}_{s}-\bar{\gamma}_{d}$ as

$\Omega(s)=\int_{0}^{s} \mathrm{w}_{s} d s-\int_{0}^{s} \bar{\gamma}_{d} d s+\Omega_{0}$

In the current context, the warping function will be determined from a weak formulation of the assumption of a constant shear flow $\bar{T}_{d}$ in the walls of the cross-section, (where $\bar{T}_{d}=G t \bar{\gamma}_{d}$ ). The strong formulation of the constraining assumption is that the contribution of the shear flow to the axial equilibrium equation, see Figure 3, of a section cut-out is zero, i.e.

$\bar{T}_{d, s}=0$

Multiplying by a virtual centerline axial displacement, $\delta \bar{u}_{z}$, and integrating over the cross-section we find the virtual work of the shear stresses in a cross-section as

$\int_{C} \bar{T}_{d, s} \delta \bar{u}_{z} d s=0$

Performing a partial integration and noting that the shear stress flow is zero at all free edges, we find the weak formulation that will be used to determine the warping function

$$
\begin{aligned}
& {\left[\bar{T}_{d} \delta \bar{u}_{z}\right]_{\text {free edges }}-\int_{C} \bar{T}_{d} \delta \bar{u}_{z, s} d s=0 \quad \Downarrow} \\
& \int_{C} \bar{T}_{d} \delta \bar{u}_{z, s} d s=0
\end{aligned}
$$

This is the constraint equation that we will use to enforce the assumption of zero axial work performed by the shear flow around the cells.

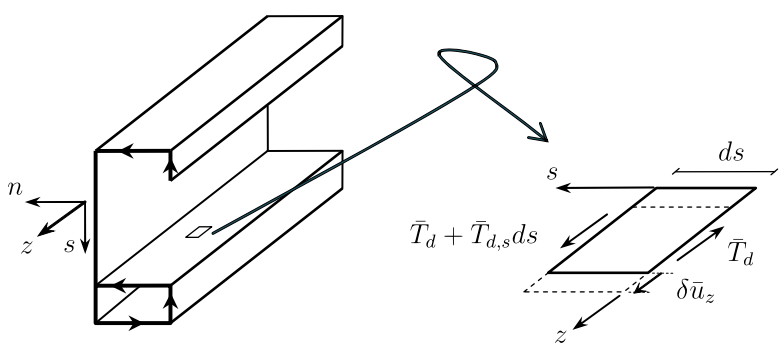

Figure 3: Work of shear flow through axial virtual displacement.

\section{Strain energy assumptions}

In the following we will adhere to simple constitutive relations, i.e. the material is assumed to be linear elastic with a modulus of elasticity $E$ and a shear modulus $G$. In the transverse direction we will assume a plate type elasticity modulus $E_{s}=E /\left(1-v^{2}\right)$, in which $v$ represents the Poisson ratio. The axial stress is determined as $\sigma=E \varepsilon$, the shear stress as $\tau=G \gamma$ and the transverse stress as $\sigma_{s}=E_{s} \varepsilon_{s}$. Thus the coupling of axial strain $\varepsilon$ and transverse strain $\varepsilon_{s}$ is neglected. Note that this means that we also neglect the equivalent coupling between axial and transverse curvatures in the constitutive relations for the plate moments, but with some changes it is possible to include the coupling of the curvatures. With the simple constitutive relations assumed, the elastic energy potential becomes

$\Pi=\int_{V}\left(\frac{1}{2} E \varepsilon^{2}+\frac{1}{2} G \gamma^{2}+\frac{1}{2} E_{s} \varepsilon_{s}^{2}\right) d V$

Let us introduce a thin-walled cross-section assembled using straight cross-sectional elements, see Figure 4, and let us integrate through the thickness, $t$, across the widths, $b_{e}$, of the elements, and over the length, $L$, of the thin-walled beam. The elastic potential energy takes the following form after the introduction of the strains expressed by the displacement in separated form

$$
\begin{aligned}
& \Pi=\int_{0}^{L}\left[\sum _ { e l } \int _ { 0 } ^ { b _ { e l } } \left\{\left[E t\left(\Omega \psi^{\prime \prime}\right)^{2}+\frac{1}{12} E t^{3}\left(\mathrm{w}_{n} \psi^{\prime \prime}\right)^{2}\right]\right.\right. \\
& +\left[G t\left(\mathrm{w}_{s} \psi^{\prime}\right)^{2}+G t\left(\Omega,{ }_{s} \psi^{\prime}\right)^{2}\right. \\
& \left.-2 G t\left(\mathrm{w}_{s} \psi^{\prime}\right)\left(\Omega,{ }_{s} \psi^{\prime}\right)+\frac{1}{3} G t^{3}\left(\mathrm{w}_{n, s} \psi^{\prime}\right)^{2}\right] \\
& \left.\left.+\left[E_{s} t\left(\mathrm{w}_{s, s} \psi\right)^{2}+\frac{1}{12} E_{s} t^{3}\left(\mathrm{w}_{n, s s} \psi\right)^{2}\right]\right\} d s\right] d z
\end{aligned}
$$

In equation (13) the elastic energy terms have been grouped in axial strain energy, shear energy, and transverse strain energy. In conventional beam theory, 


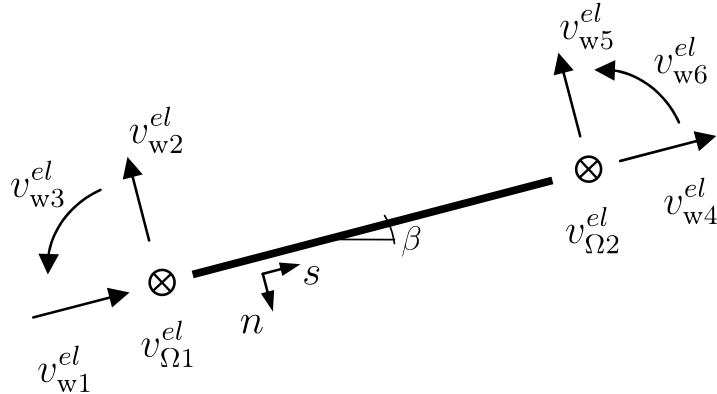

Figure 4: Components of the displacements vectors of a straight crosssection element.

we usually introduce rigid cross-sectional displacement modes and the elastic energy is described by a summation of the energy stored in all displacement modes. However, we have to remember the shear constraints associated with our assumption of constant shear flow, which have to be introduced later. In the current work we wish to establish a set of displacement modes by using semi discretization. To achieve this, the crosssection will be divided into discrete straight-line elements, in which we interpolate the transverse and axial displacements.

\section{Interpolation within cross-section elements}

Within each straight finite cross-section element, the axial displacements, $\Omega$, will be interpolated linearly corresponding to a linear variation of the warping functions and the transverse displacement of the elements will be interpolated linearly in the direction of the element and cubically (corresponding to beam elements) in the transverse direction of the element. The displacements in a straight cross-section finite element are thus interpolated as follows:

$$
\begin{aligned}
\Omega \psi^{\prime} & =\mathbf{N}_{\Omega} \mathbf{v}_{\Omega}^{e l} \psi^{\prime} \\
\mathrm{w}_{s} \psi & =\mathbf{N}_{s} \mathbf{v}_{\mathrm{w}}^{e l} \psi \\
\mathrm{w}_{n} \psi & =\mathbf{N}_{n} \mathbf{v}_{\mathrm{w}}^{e l} \psi
\end{aligned}
$$

in which $\mathbf{N}_{\Omega}(s)$ and $\mathbf{N}_{s}(s)$ are linear interpolation matrices and $\mathbf{N}_{n}(s)$ is a cubic (beam) interpolation matrix. Furthermore we have introduced the axial and transverse nodal displacement components of a straight

$$
\begin{aligned}
\mathbf{k}_{\Omega \Omega}^{\sigma} & =\int_{0}^{b_{e}} E t \mathbf{N}_{\Omega}^{T} \mathbf{N}_{\Omega} d s \\
\mathbf{k}_{\mathrm{ww}}^{\sigma} & =\int_{0}^{b_{e}} \frac{E t^{3}}{12} \mathbf{N}_{n}^{T} \mathbf{N}_{n} d s \\
\mathbf{k}^{s} & =\int_{0}^{b_{e}}\left(E_{s} t \mathbf{N}_{s, s}^{T} \mathbf{N}_{s, s}+\frac{E_{s} t^{3}}{12} \mathbf{N}_{n, s s}^{T} \mathbf{N}_{n, s s}\right) d s \\
\mathbf{k}_{\mathrm{ww}}^{\tau} & =\int_{0}^{b_{e}}\left(G t \mathbf{N}_{s}^{T} \mathbf{N}_{s}+\frac{G t^{3}}{3} \mathbf{N}_{n, s}^{T} \mathbf{N}_{n, s}\right) d s \\
\mathbf{k}_{\Omega \Omega}^{\tau} & =\int_{0}^{b_{e}} G t \mathbf{N}_{\Omega, s}^{T} \mathbf{N}_{\Omega, s} d s \\
\mathbf{k}_{\mathrm{w} \Omega}^{\tau} & =\left[\mathbf{k}_{\Omega \mathrm{w}}^{\tau}\right]^{T}=-\int_{0}^{b_{e}} G t \mathbf{N}_{s}^{T} \mathbf{N}_{\Omega, s} d s
\end{aligned}
$$

Table 1: Straight-element stiffness contributions.

$$
\begin{array}{rlrl}
\mathbf{K}_{\Omega \Omega}^{\sigma} & =\sum_{e l} \mathbf{T}_{\Omega}^{T} \mathbf{k}_{\Omega \Omega}^{\sigma} \mathbf{T}_{\Omega} & \mathbf{K}_{\mathrm{ww}}^{\tau} & =\sum_{e l} \mathbf{T}_{\mathrm{w}}^{T} \mathbf{k}_{\mathrm{ww}}^{\tau} \mathbf{T}_{\mathrm{w}} \\
\mathbf{K}_{\mathrm{ww}}^{\sigma}=\sum_{e l}^{e l} \mathbf{T}_{\mathrm{w}}^{T} \mathbf{k}_{\mathrm{ww}}^{\sigma} \mathbf{T}_{\mathrm{w}} & \mathbf{K}_{\Omega \Omega}^{\tau}=\sum_{e l} \mathbf{T}_{\Omega}^{T} \mathbf{k}_{\Omega \Omega}^{\tau} \mathbf{T}_{\Omega} \\
\mathbf{K}^{s}=\sum_{e l} \mathbf{T}_{\mathrm{w}}^{T} \mathbf{k}^{s} \mathbf{T}_{\mathrm{w}} & \mathbf{K}_{\mathrm{w} \Omega}^{\tau}=\sum_{e l} \mathbf{T}_{\mathrm{w}}^{T} \mathbf{k}_{\mathrm{w} \Omega}^{\tau} \mathbf{T}_{\Omega}
\end{array}
$$

Table 2: Assembly into total cross-section stiffness contributions.

\section{cross-section element as}

$$
\begin{aligned}
& \mathbf{v}_{\Omega}^{e l}=\left[\begin{array}{ll}
v_{\Omega 1}^{e l} & v_{\Omega 2}^{e l}
\end{array}\right]^{T} \\
& =\left[\begin{array}{ll}
\Omega(0) & \Omega\left(b_{e}\right)
\end{array}\right]^{T} \\
& \mathbf{v}_{\mathrm{w}}^{e l}=\left[\begin{array}{llllll}
v_{\mathrm{w} 1}^{e l} & v_{\mathrm{w} 2}^{e l} & v_{\mathrm{w} 3}^{e l} & v_{\mathrm{w} 4}^{e l} & v_{\mathrm{w} 5}^{e l} & v_{\mathrm{w} 6}^{e l}
\end{array}\right]^{T} \\
& =\left[\begin{array}{lll}
\mathrm{w}_{s}(0) & -\mathrm{w}_{n}(0) & -\mathrm{w}_{n, s}(0)
\end{array}\right. \\
& \left.\mathrm{w}_{s}\left(b_{e}\right)-\mathrm{w}_{n}\left(b_{e}\right) \quad-\mathrm{w}_{n, s}\left(b_{e}\right)\right]^{T}
\end{aligned}
$$

Here $b_{e}$ is the width of the flat element. Nodal components and the direction of the section coordinates $(n, s)$ are shown in Figure 4. The element stiffness contributions to the axial strain, shear strain, and transverse strain energy can now be found using the displacement interpolations. The stiffness contributions found are shown in Table 1, in which the first two are the axial stiffness contributions, the third is the transverse distortional stiffness term, while the last three are the shear strain stiffness contributions. These stiffness contributions can be found explicitly. Let us prepare for the formulation of the total cross-section elastic energy by introducing global displacement vectors as an assembly of the local element degrees of freedom. The axial displacements and the transverse displacements are sepa- 
rated into two vectors as follows:

$$
\begin{aligned}
\mathbf{v}_{\Omega} & =\left[\begin{array}{lllllll}
v_{\Omega 1} & v_{\Omega 2} & v_{\Omega 3} & \ldots
\end{array}\right]^{T} \\
\mathbf{v}_{\mathrm{w}} & =\left[\begin{array}{lllllll}
v_{x 1} & v_{y 1} & \phi_{1} & v_{x 2} & v_{y 2} & \phi_{2} & \ldots
\end{array}\right]^{T}
\end{aligned}
$$

In equation (16), $\mathbf{v}_{\Omega}$ holds the local axial element degrees of freedom, and $\mathbf{v}_{\mathrm{w}}$ holds the local element degrees of freedom, corresponding to two displacements and one rotation for each node. The transformation from local to global components is performed using a formal standard transformation of the components in the crosssection plane, i.e. $\mathbf{v}_{\Omega}=\mathbf{T}_{\Omega} \mathbf{v}_{\Omega}^{e l}$ and $\mathbf{v}_{\mathrm{w}}=\mathbf{T}_{\mathrm{w}} \mathbf{v}_{\mathrm{w}}^{e l}$. The global assembly of stiffness matrices is found by summation of the contribution from each element, as illustrated in Table 2. Introducing the described interpolation and matrix calculation scheme, the elastic potential energy in equation (13) now takes the following form:

$$
\begin{aligned}
\Pi=\frac{1}{2} \int_{0}^{L}\left\{\left[\begin{array}{ll}
\psi \mathbf{v}_{\mathrm{w}}^{T} & \psi \mathbf{v}_{\Omega}^{T}
\end{array}\right]^{\prime \prime}\left[\begin{array}{cc}
\mathbf{K}_{\mathrm{ww}}^{\sigma} & \mathbf{0} \\
\mathbf{0} & \mathbf{K}_{\Omega \Omega}^{\sigma}
\end{array}\right]\left[\begin{array}{l}
\psi \mathbf{v}_{\mathrm{w}} \\
\psi \mathbf{v}_{\Omega}
\end{array}\right]^{\prime \prime}\right. \\
+\left[\begin{array}{ll}
\psi \mathbf{v}_{\mathrm{w}}^{T} & \psi \mathbf{v}_{\Omega}^{T}
\end{array}\right]^{\prime}\left[\begin{array}{cc}
\mathbf{K}_{\mathrm{ww}}^{\tau} & \mathbf{K}_{\mathrm{w} \Omega}^{\tau} \\
\mathbf{K}_{\Omega \mathrm{w}}^{\tau} & \mathbf{K}_{\Omega \Omega}^{\tau}
\end{array}\right]\left[\begin{array}{l}
\psi \mathbf{v}_{\mathrm{w}} \\
\psi \mathbf{v}_{\Omega}
\end{array}\right]^{\prime} \\
\left.+\left[\begin{array}{ll}
\psi \mathbf{v}_{\mathrm{w}}^{T} & \psi \mathbf{v}_{\Omega}^{T}
\end{array}\right]\left[\begin{array}{cc}
\mathbf{K}^{s} & \mathbf{0} \\
\mathbf{0} & \mathbf{0}
\end{array}\right]\left[\begin{array}{l}
\psi \mathbf{v}_{\mathrm{w}} \\
\psi \mathbf{v}_{\Omega}
\end{array}\right]\right\} d z
\end{aligned}
$$

In equation (17) and in the following, a bold zero $\mathbf{0}$ denotes a suitable size matrix or vector of zeroes. The axial stiffness from transverse displacements sub matrix $\mathbf{K}_{\mathrm{ww}}^{\sigma}$ has a rank deficiency equal to the number of free end nodes plus the number of "internal" nodes between corner points of the cross-section. The in-plane crosssection distortional stiffness sub matrix $\mathbf{K}^{s}$ has a rank deficiency of 3, corresponding to three in-plane "rigid body" or rather non-distortional displacements of the cross-section. Finally the whole shear stiffness matrix has a rank deficiency of 3 , corresponding to the existence of pure axial extension and two pure flexural modes without shear. It turns out that since the pure axial displacement only involves the sub matrix $\mathbf{K}_{\Omega \Omega}^{\tau}$, this matrix has a rank deficiency of one.

\section{Constraints, eliminations, transformations and reduction of order}

To achieve a formulation resembling a generalization of Vlasov beam theory including distortion, the following three main steps need to be performed, before we can solve the eigenvalue problem related to distortional displacements, including local plate type modes. In this process, we introduce constraints, and we identify and eliminate the basic solutions related to the conventional beam displacement modes.

\subsection{Step I-Pure axial extension and shear constraints}

In this step, we introduce the shear constraint equations that bind axial and transverse modes together and at the same time simplify or condense equation (17). In this process we need to eliminate the singularity in the shear stiffness matrix related to pure axial extension. The first eigenmode that we identify is the pure axial extension; it produces no shear energy and no transverse displacement energy (due to the simple constitutive relations assumed, corresponding to beam theory and the mentioned rank deficiency).

Let us introduce the shear constraint equations using equation (11) as follows:

$$
\begin{aligned}
& \int_{C} \bar{T}_{d} \delta \bar{u}_{z, s} d s=0 \quad \Downarrow \\
& -\int_{C}\left(G t\left(\mathrm{w}_{s}-\Omega,{ }_{s}\right)\right) \delta \Omega,{ }_{s} d s=0 \quad \Downarrow \\
& \int_{C} G t \Omega,{ }_{s} \delta \Omega,,_{s} d s=\int_{C} G t \mathrm{w}_{s} \delta \Omega,{ }_{s} d s
\end{aligned}
$$

Introducing the interpolation, see equation (14), Tables 1 and 2, and taking variations gives us the constraint equations

$\mathbf{K}_{\Omega \Omega}^{\tau} \mathbf{v}_{\Omega}=-\mathbf{K}_{\Omega \mathrm{w}}^{\tau} \mathbf{v}_{\mathrm{W}}$

This matrix equation is singular because pure axial extension does not produce shear. Therefore we introduce the following transformation, using superscripts $a$ for axial and $o$ for other:

$\mathbf{v}_{\Omega}=\left[\begin{array}{ll}\mathbf{T}_{\Omega}^{a} & \mathbf{T}_{\Omega}^{o}\end{array}\right]\left[\begin{array}{l}v_{\Omega}^{a} \\ \mathbf{v}_{\Omega}^{o}\end{array}\right]$

in which $\mathbf{T}_{\Omega}^{a}=\mathbf{v}_{\Omega}^{\text {axial }}$ is the pure axial deformation mode (with unit value for all components). The other remaining modes are picked out by

$\left.\mathbf{T}_{\Omega}^{o}=\left[\begin{array}{c}\mathbf{0} \\ \mathbf{I}_{a a}\end{array}\right]\right\} \begin{aligned} & 1 \text { d.o.f. related to the first node } \\ & \text { Unit diagonal matrix }\end{aligned}$

Here $\mathbf{I}_{a a}$ is a unit diagonal matrix of dimension $n_{a}=$ $n_{n o}-1$, where $n_{n o}$ is the number of nodes. Furthermore, it is worth noting that $v_{\Omega}^{a}$ is one component that corresponds to the amount of pure axial extension, while $\mathbf{v}_{\Omega}^{o}$ corresponds to all the other axial displacement degrees of freedom. Introducing the transformation in equation (20) into the constraint equations (19), we get the following by pre- and post multiplication:

$\left[\begin{array}{cc}0 & \mathbf{0} \\ \mathbf{0} & \mathbf{T}_{\Omega}^{o T} \mathbf{K}_{\Omega \Omega}^{\tau} \mathbf{T}_{\Omega}^{o}\end{array}\right]\left[\begin{array}{c}v_{\Omega}^{a} \\ \mathbf{v}_{\Omega}^{o}\end{array}\right]=\left[\begin{array}{c}0 \\ -\mathbf{T}_{\Omega}^{o T} \mathbf{K}_{\Omega \mathrm{w}}^{\tau} \mathbf{v}_{\mathrm{W}}\end{array}\right] \Downarrow$

$\mathbf{v}_{\Omega}^{o}=-\left(\mathbf{K}_{\Omega \Omega}^{\tau o o}\right)^{-1} \mathbf{K}_{\Omega \mathrm{w}}^{\tau o} \mathbf{v}_{\mathrm{w}}$ 
where the matrices $\mathbf{K}_{\Omega \Omega}^{\tau o o}$ and $\mathbf{K}_{\Omega \mathrm{w}}^{\tau o}$ are given in Table 3 . With equation (22) we have introduced a transformation from in-plane cross-section displacement modes to the axial displacement modes without pure axial extension as follows:

$\mathbf{v}_{\Omega}^{o}=\mathbf{T}_{\Omega \mathrm{w}} \mathbf{v}_{\mathrm{w}}$, where $\mathbf{T}_{\Omega \mathrm{w}}=-\left(\mathbf{K}_{\Omega \Omega}^{\tau o o}\right)^{-1} \mathbf{K}_{\Omega \mathrm{w}}^{\tau o}$

Combining equation (20) and (22) gives

$$
\begin{aligned}
\mathbf{v}_{\Omega} & =\left[\begin{array}{ll}
\mathbf{T}_{\Omega}^{o} \mathbf{T}_{\Omega \mathrm{w}} & \mathbf{T}_{\Omega}^{a}
\end{array}\right]\left[\begin{array}{c}
\mathbf{v}_{\mathrm{W}} \\
v_{\Omega}^{a}
\end{array}\right]=\left[\begin{array}{ll}
\mathbf{T}_{\Omega \mathrm{w}}^{r} & \mathbf{T}_{\Omega}^{a}
\end{array}\right]\left[\begin{array}{c}
\mathbf{v}_{\mathrm{W}} \\
v_{\Omega}^{a}
\end{array}\right] \\
& =\mathbf{T}_{\Omega \mathrm{w}}^{r} \mathbf{v}_{\mathrm{W}}+\mathbf{T}_{\Omega}^{a} v_{\Omega}^{a}
\end{aligned}
$$

where $\mathbf{T}_{\Omega \mathrm{w}}^{r}=\mathbf{T}_{\Omega}^{o} \mathbf{T}_{\Omega \mathrm{w}}$.

The potential energy formulation (17) can now be modified so that the amount of axial extension is described by the separate degree of freedom $v_{\Omega}^{a}$ and the shear constraint equations are enforced. The modification of (17) is performed using the transformation in equation (24) and, to clarify the variational treatment of pure axial extension, we also temporally rewrite the terms pertaining to axial extension using $\zeta v_{\Omega}^{a}=-\psi^{\prime} v_{\Omega}^{a}$. Introducing transformed stiffness matrices, see Table 3 , the elastic potential energy (for one mode) takes the following form

$$
\begin{aligned}
& \Pi= \\
& \frac{1}{2} \int_{0}^{L}\left\{\left[\begin{array}{ll}
\left(\psi \mathbf{v}_{\mathrm{w}}^{T}\right)^{\prime \prime} & \left(\zeta v_{\Omega}^{a} T\right)^{\prime}
\end{array}\right]\left[\begin{array}{cc}
\overline{\mathbf{K}}^{\sigma} & -\mathbf{K}_{\Omega \Omega}^{\sigma r a} \\
-\mathbf{K}_{\Omega \Omega}^{\sigma a r} & K_{\Omega \Omega}^{\sigma a a}
\end{array}\right]\left[\begin{array}{c}
\left(\psi \mathbf{v}_{\mathrm{w}}\right)^{\prime \prime} \\
\left(\zeta v_{\Omega}^{a}\right)^{\prime}
\end{array}\right]\right. \\
& \left.+\left(\psi \mathbf{v}_{\mathrm{w}}^{T}\right)^{\prime} \mathbf{K}^{\tau}\left(\psi \mathbf{v}_{\mathrm{w}}\right)^{\prime}+\left(\psi \mathbf{v}_{\mathrm{w}}^{T}\right) \mathbf{K}^{s}\left(\psi \mathbf{v}_{\mathrm{w}}\right)\right\} d z
\end{aligned}
$$

To find the homogeneous distortional differential equations of GBT, the first variation of the elastic potential energy is investigated by taking variations in the complete displacement field. The virtual variation of a property is denoted by a $\delta$ in front of the varied field property (displacement field), as in $\delta\left(v_{\mathrm{w}} \psi\right)^{\prime}$, as the virtual variation of the first derivative of the transverse displacement field expressed by the product of the transverse displacement shape $\mathbf{v}_{\mathrm{w}}$ and the axial variation $\psi^{\prime}$. This gives us

$$
\begin{aligned}
& \delta \Pi= \\
& \int_{0}^{L}\left\{\delta\left(\psi \mathbf{v}_{\mathrm{w}}^{T}\right)^{\prime \prime}\left[\overline{\mathbf{K}}^{\sigma}\left(\psi \mathbf{v}_{\mathrm{w}}\right)^{\prime \prime}-\mathbf{K}_{\Omega \Omega}^{\sigma r a}\left(\zeta v_{\Omega}^{a}\right)^{\prime}\right]\right. \\
& \quad+\delta\left(\psi \mathbf{v}_{\mathrm{w}}^{T}\right)^{\prime} \mathbf{K}^{\tau}\left(\psi \mathbf{v}_{\mathrm{w}}\right)^{\prime}+\delta\left(\psi \mathbf{v}_{\mathrm{w}}^{T}\right) \mathbf{K}^{s}\left(\psi \mathbf{v}_{\mathrm{w}}\right) \\
& \left.\quad+\delta\left(\zeta v_{\Omega}^{a}\right)^{\prime}\left[-K_{\Omega \Omega}^{\sigma a r}\left(\psi \mathbf{v}_{\mathrm{w}}\right)^{\prime \prime}+K_{\Omega \Omega}^{\sigma a a}\left(\zeta v_{\Omega}^{a}\right)^{\prime}\right]\right\} d z
\end{aligned}
$$

After performing up to two partial integrations on the terms and derived terms that involve axial derivatives of the (virtual) varied displacement field, $\delta()^{\prime}$ or $\delta()^{\prime \prime}$, the first variation of the elastic potential energy takes the form:

$$
\begin{aligned}
& \delta \Pi= \\
& \begin{array}{c}
\int_{0}^{L}\left\{\delta ( \psi \mathbf { v } _ { \mathrm { w } } ^ { T } ) \left[\overline{\mathbf{K}}^{\sigma} \mathbf{v}_{\mathrm{w}} \psi^{\prime \prime \prime \prime}-\mathbf{K}_{\Omega \Omega}^{\sigma r a} v_{\Omega}^{a} \zeta^{\prime \prime \prime}\right.\right. \\
\left.-\mathbf{K}^{\tau} \mathbf{v}_{\mathrm{w}} \psi^{\prime \prime}+\mathbf{K}^{s} \mathbf{v}_{\mathrm{w}} \psi\right] \\
\left.+\delta\left(\zeta v_{\Omega}^{a}\right)\left[\mathbf{K}_{\Omega \Omega}^{\sigma a r} \mathbf{v}_{\mathrm{w}} \psi^{\prime \prime \prime}-K_{\Omega \Omega}^{\sigma a a} v_{\Omega}^{a} \zeta^{\prime \prime}\right]\right\} d s \\
+\left[\delta\left(\psi \mathbf{v}_{\mathrm{w}}^{T}\right)^{\prime}\left[\overline{\mathbf{K}}^{\sigma}\left(\psi \mathbf{v}_{\mathrm{w}}\right)^{\prime \prime}-\mathbf{K}_{\Omega \Omega}^{\sigma r a}\left(\zeta v_{\Omega}^{a}\right)^{\prime}\right]\right. \\
+\delta\left(\psi \mathbf{v}_{\mathrm{w}}^{T}\right)\left[-\overline{\mathbf{K}}^{\sigma}\left(\psi \mathbf{v}_{\mathrm{w}}\right)^{\prime \prime \prime}+\mathbf{K}_{\Omega \Omega}^{\sigma r a}\left(\zeta v_{\Omega}^{a}\right)^{\prime \prime}+\mathbf{K}^{\tau}\left(\psi \mathbf{v}_{\mathrm{w}}\right)^{\prime}\right] \\
\left.+\delta\left(\zeta v_{\Omega}^{a}\right)\left[-\mathbf{K}_{\Omega \Omega}^{\sigma a r}\left(\psi \mathbf{v}_{\mathrm{w}}\right)^{\prime \prime}-K_{\Omega \Omega}^{\sigma a a}\left(\zeta v_{\Omega}^{a}\right)^{\prime}\right]\right]_{0}^{L}
\end{array}
\end{aligned}
$$

For internal variation in the displacement fields $\delta\left(\psi \mathbf{v}_{\mathrm{W}}\right)$ and $\delta\left(\zeta v_{\Omega}^{a}\right)$, the elastic potential energy should be stationary and therefore its first variation must be equal to zero. Here the terms in the squared bracket correspond to the boundary loads and boundary conditions. Taking internal variations reveals the following coupled homogeneous differential equations of GBT in which we note that $\zeta=-\psi^{\prime}$ :

$$
\begin{aligned}
& \overline{\mathbf{K}}^{\sigma} \mathbf{v}_{\mathrm{w}} \psi^{\prime \prime \prime \prime}-\mathbf{K}_{\Omega \Omega}^{\sigma r a} v_{\Omega}^{a} \zeta^{\prime \prime \prime}-\mathbf{K}^{\tau} \mathbf{v}_{\mathrm{w}} \psi^{\prime \prime}+\mathbf{K}^{s} \mathbf{v}_{\mathrm{w}} \psi=\mathbf{0} \\
& \mathbf{K}_{\Omega \Omega}^{\sigma a r} \mathbf{v}_{\mathrm{w}} \psi^{\prime \prime \prime}-K_{\Omega \Omega}^{\sigma a a} v_{\Omega}^{a} \zeta^{\prime \prime}=\mathbf{0}
\end{aligned}
$$

These equations establish a coupled set of homogeneous GBT differential equations that determine the displacements of a thin-walled beam for a given set of boundary conditions. To solve the boundary value problem, it is necessary to solve the related eigenvalue problem by establishing the eigenmodes/vectors and the related axial variation through the related eigenvalues. In the following, we will first consider the case where the displacement vectors do not contain transverse displacements, and then we will consider one in which they do. We start out by isolating the term $v_{\Omega}^{a} \zeta^{\prime \prime}$ in equation (29) as follows:

$v_{\Omega}^{a} \zeta^{\prime \prime}=\left(K_{\Omega \Omega}^{\sigma a a}\right)^{-1} \mathbf{K}_{\Omega \Omega}^{\sigma a r} \mathbf{v}_{\mathrm{w}} \psi^{\prime \prime \prime}$

We can identify pure axial extension as an eigenmode solution. For the above equations (28) and (29), it corresponds to $\left(\mathbf{v}_{\mathrm{w}}, v_{\Omega}^{a}\right)=(\mathbf{0}, 1)$, which we can see leads 


\begin{tabular}{ll}
\hline$K_{\Omega \Omega}^{\sigma a a}=\mathbf{T}_{\Omega}^{a T} \mathbf{K}_{\Omega \Omega}^{\sigma} \mathbf{T}_{\Omega}^{a}$ & $\mathbf{K}_{\Omega \Omega}^{\tau r r}=\mathbf{T}_{\Omega \mathrm{w}}^{r}{ }^{T} \mathbf{K}_{\Omega \Omega}^{\tau} \mathbf{T}_{\Omega \mathrm{w}}^{r}$ \\
$\mathbf{K}_{\Omega \Omega}^{\sigma a r}=\mathbf{T}_{\Omega}^{a T} \mathbf{K}_{\Omega \Omega}^{\sigma} \mathbf{T}_{\Omega \mathrm{w}}^{r}$ & $\mathbf{K}_{\mathrm{w} \Omega}^{\tau r}=K_{\mathrm{w} \Omega}^{\tau} \mathbf{T}_{\Omega \mathrm{w}}^{r}=\mathbf{K}_{\Omega \mathrm{w}}^{\tau r}{ }^{r}$ \\
$\mathbf{K}_{\Omega \Omega}^{\sigma r r}=\mathbf{T}_{\Omega \mathrm{w}}^{r}{ }^{T} \mathbf{K}_{\Omega \Omega}^{\sigma} \mathbf{T}_{\Omega \mathrm{w}}^{r}$ & $\mathbf{K}_{\Omega \Omega}^{\tau o o}=\mathbf{T}_{\Omega}^{o T} \mathbf{K}_{\Omega \Omega}^{\tau} \mathbf{T}_{\Omega}^{o}$ \\
$\overline{\mathbf{K}}^{\sigma}=\mathbf{K}_{\mathrm{ww}}^{\sigma}+\mathbf{K}_{\Omega \Omega}^{\sigma r r}$ & $\mathbf{K}_{\Omega \mathrm{w}}^{\tau o}=\mathbf{T}_{\Omega}^{o T} \mathbf{K}_{\Omega \mathrm{w}}^{\tau}$ \\
$\mathbf{K}^{\sigma}=\overline{\mathbf{K}}^{\sigma}-\mathbf{K}_{\Omega \Omega}^{\sigma r a}\left(K_{\Omega \Omega}^{\sigma a a}\right)^{-1} \mathbf{K}_{\Omega \Omega}^{\sigma a r}$ & $\mathbf{K}^{\tau}=\mathbf{K}_{\mathrm{ww}}^{\tau}+\mathbf{K}_{\mathrm{w} \Omega}^{\tau r}+\mathbf{K}_{\Omega \mathrm{w}}^{\tau r}+\mathbf{K}_{\Omega \Omega}^{\tau r r}$ \\
\hline
\end{tabular}

Table 3: Transformation of stiffness matrices related to Step I.

to a solution. In the original "global" space, the axial eigenmode is given by $\left(\mathbf{v}_{\mathrm{w}}, \mathbf{v}_{\Omega}\right)=\left(\mathbf{0}, \mathbf{T}_{\Omega}^{a}\right)$. It is also clear that the axial variation of pure axial extension can be determined by double integration of equation (30) with $\mathbf{v}_{\mathrm{w}}=\mathbf{0}$, which results in:

$$
\begin{aligned}
\zeta(z) & =-\psi^{\prime}(z)=c_{a 1}+c_{a 2} z \\
& =-\Psi_{a}^{\prime}(z) \mathbf{c}_{a}=\left[\begin{array}{ll}
1 & z
\end{array}\right]\left[\begin{array}{l}
c_{a 1} \\
c_{a 2}
\end{array}\right]
\end{aligned}
$$

where $c_{a 1}$ and $c_{a 2}$ are constants determined by the boundary conditions of axial extension.

Having identified the "trivial" eigenmode, pure axial extension, we finally turn to the solution of the transverse displacement modes. In this case, equation (30) determines the correction term that eliminates pure axial extension in the back substitution process. Eliminating $\zeta^{\prime \prime}$ by using the fact that $\zeta^{\prime \prime}=-\psi^{\prime \prime \prime}$ and assuming that $\psi^{\prime \prime \prime} \neq 0$, we find:

$v_{\Omega}^{a}=-\left(K_{\Omega \Omega}^{\sigma a a}\right)^{-1} \mathbf{K}_{\Omega \Omega}^{\sigma a r} \mathbf{v}_{\mathrm{W}}$

Using this equation or equation (29), we eliminate the second term in equation (28) and introduce $\mathbf{K}^{\sigma}$, as given in Table 3. This results in the following homogeneous fourth order differential equations for determination of the transverse (global, distortional and local) distortional displacement modes of GBT:

$\mathbf{K}^{\sigma} \mathbf{v}_{\mathrm{w}} \psi^{\prime \prime \prime \prime}-\mathbf{K}^{\tau} \mathbf{v}_{\mathrm{w}} \psi^{\prime \prime}+\mathbf{K}^{s} \mathbf{v}_{\mathrm{w}} \psi=\mathbf{0}$

To solve this set of equations we have to solve the related eigenvalue problem, which is of 4th order, but since only an even number of axial derivatives is involved, this reduces to a quadratic eigenvalue problem. With solutions, $\mathbf{v}_{\mathrm{w}}$, to equation (33), we can find $\mathbf{v}_{\Omega}^{o}$ using equation (23), $v_{\Omega}^{a}$ using equation (32), and finally $\mathbf{v}_{\Omega}$ using equation (20), thus revealing the full solution (in global space).

\subsection{Step II - Rigid cross-section displacements and constant wall-width constraint}

In this step, we will identify and eliminate two eigenmodes corresponding to transverse translation of the cross-section, and we will identify a pure rotational eigenmode for later elimination in the next step. Furthermore, we will also constrain the transverse displacement field, so that the wall widths remain constant, i.e. we will enforce $\mathrm{w}_{s, s} \equiv 0$, see equation (5).

With the introduction of the shear constraints in the previous step, the flexural modes do not have shear energy and the shear stiffness matrix $\mathbf{K}^{\tau}$ is therefore singular for these modes. Since neither the pure translational modes nor the rotational mode involve any distortion of the cross-section, the transverse stiffness matrix $\mathbf{K}^{s}$ will be singular for these modes. It turns out that the translational modes correspond to two quadruple zero eigenvalues or roots of the related characteristic equation. To orthogonalize these modes with respect to the non-singular axial stiffness matrix $\mathbf{K}^{\sigma}$, we will form the subspace spanned by the modes and orthogonalize in this subspace.

Let us first introduce two not necessarily orthogonal translational modes corresponding to a unit translation in each transverse coordinate direction, ordered in columns in the matrix $\mathbf{T}_{\mathrm{w}}^{x y}=\left[\mathbf{v}_{\mathrm{w}}^{\mathrm{x} \text { trans }} \quad \mathbf{v}_{\mathrm{w}}^{\mathrm{y} \text { trans }}\right]$ and a rotational mode corresponding to rotation about the origin of the (initial) transverse coordinate axes $\mathbf{T}_{\mathrm{w}}^{\mathrm{z}}=\left[\mathbf{v}_{\mathrm{w}}^{\mathrm{z} \text { rot }}\right]$.

In the subspace spanned by the two non-orthogonal translational modes introduced, we can find the principle flexural directions by an equivalent conventional method or by finding the eigenvectors of the following two-dimensional eigenvalue problem:

$\left(\mathbf{K}_{x y}^{\sigma}-\lambda \mathbf{I}\right) \mathbf{v}_{x y}=\mathbf{0} \quad \Rightarrow \quad \mathbf{v}_{x y}=\mathbf{v}_{x y}^{1}$ or $\mathbf{v}_{x y}^{2}$

where $\mathbf{I}$ is a $2 \times 2$ diagonal unit matrix and $\mathbf{K}_{x y}^{\sigma}=$ $\mathbf{T}_{\mathrm{w}}^{x y T} \mathbf{K}^{\sigma} \mathbf{T}_{\mathrm{w}}^{x y}$. The two orthogonal eigenvectors corresponding to the principle axis directions are ordered in columns in the transformation matrix

$\mathbf{T}_{x y}^{\alpha}=\left[\begin{array}{ll}\mathbf{v}_{x y}^{1} & \mathbf{v}_{x y}^{2}\end{array}\right]$

Finally we can determine the two orthogonal translational eigenmodes in the full $\mathbf{v}_{\mathrm{w}}$ space, ordered in columns in a transformation matrix as $\mathbf{T}_{\mathrm{w}}^{\alpha}=$ 
$\left[\mathbf{v}_{\mathrm{w}}^{1 \text { trans }} \mathbf{v}_{\mathrm{w}}^{2 \text { trans }}\right]$, by the simple matrix multiplication

$\mathbf{T}_{\mathrm{w}}^{\alpha}=\mathbf{T}_{\mathrm{w}}^{x y} \mathbf{T}_{x y}^{\alpha}$

Next we turn to the non-orthogonal rotational mode, and we subtract the translational part, so that the coupling term in the axial stiffness vanishes. Thus the orthogonal pure rotational mode is given by

$\mathbf{v}_{\mathrm{w}}^{3 \text { rot }}=\mathbf{v}_{\mathrm{w}}^{\mathrm{z} \text { rot }}-\left[\begin{array}{ll}\mathbf{v}_{\mathrm{w}}^{1 \text { trans }} & \mathbf{v}_{\mathrm{w}}^{2 \text { trans }}\end{array}\right] \mathbf{d}_{\alpha} \quad \mathbb{i}$

$\mathbf{T}_{\mathrm{w}}^{3}=\mathbf{T}_{\mathrm{w}}^{z}-\mathbf{T}_{\mathrm{w}}^{\alpha} \mathbf{d}_{\alpha}$

where we have introduced the transformation "matrix" $\mathbf{T}_{\mathrm{w}}^{3}=\left[\mathbf{v}_{\mathrm{w}}^{3 \text { rot }}\right]$ and $\mathbf{d}_{\alpha}$ as a two-dimensional vector giving the amount of each translational eigenmode to be subtracted. Note that $\mathbf{d}_{\alpha}$ is related to the coordinate vector of the shear center, see [12]. The coupling terms in the axial stiffness between translations and rotation are found as follows (in the subspace):

$$
\begin{aligned}
\mathbf{K}_{\alpha 3}^{\sigma} & =\mathbf{T}_{\mathrm{w}}^{\alpha T} \mathbf{K}^{\sigma} \mathbf{T}_{\mathrm{w}}^{3} \\
& =\mathbf{T}_{\mathrm{w}}^{\alpha T} \mathbf{K}^{\sigma}\left(\mathbf{T}_{\mathrm{w}}^{z}-\mathbf{T}_{\mathrm{w}}^{\alpha} \mathbf{d}_{\alpha}\right) \\
& =\mathbf{K}_{\alpha z}^{\sigma}-\mathbf{K}_{\alpha \alpha}^{\sigma} \mathbf{d}_{\alpha}
\end{aligned}
$$

By requiring that the coupling terms in the axial stiffness vanish $\mathbf{K}_{\alpha 3}^{\sigma}=\mathbf{0}$, we find

$\mathbf{d}_{\alpha}=\mathbf{K}_{\alpha \alpha}^{\sigma-1} \mathbf{K}_{\alpha \mathbf{z}}^{\sigma}$

Now we can completely identify the orthogonal pure rotational eigenmode by inserting equation (39) in (37) as

$\mathbf{T}_{\mathrm{w}}^{3}=\mathbf{T}_{\mathrm{w}}^{z}-\mathbf{T}_{\mathrm{w}}^{\alpha} \mathbf{K}_{\alpha \alpha}^{\sigma-1} \mathbf{K}_{\alpha z}^{\sigma}$

Here the matrix transformations are given in Table 4.

Before performing eliminations and finding the solutions pertaining to the translational modes, we will constrain the transverse normal strains in the middle surface of the cross-section walls, i.e. we will enforce $\mathrm{w}_{s, s} \equiv 0$ or, say, enforce a constant wall-width constraint. For each wall element, this leads to a multi-point constraint equation in local degrees of freedom, $\mathbf{v}_{\mathrm{w}}^{e l}$, corresponding to no centerline elongation. It takes the following form:

$$
\left[\begin{array}{llllll}
1 & 0 & 0 & -1 & 0 & 0
\end{array}\right] \mathbf{v}_{\mathrm{w}}^{e l}=0
$$

Each element constraint equation is reformulated into global degrees of freedom by a formal transformation of the form $\mathbf{v}_{\mathrm{w}}^{e l}=\mathbf{T}_{\mathrm{w}}^{T} \mathbf{v}_{\mathrm{w}}$, which allows us to write $n_{c}$ independent constraint equations (where $n_{c}$ can be less than the number of elements due to over-constraining). The equations take the following form in the full $\mathbf{v}_{\mathrm{w}^{-}}$ space:

$$
\mathbf{C v}_{\mathrm{W}}=\mathbf{0}
$$

The transformation method described by Cook et al. [26] will be used to enforce the multi-point constraint equations and eliminate the related degrees of freedom. However, we must also incorporate the elimination of the translational eigenmodes and prepare for the elimination of the rotational modes.

Before any elimination of eigenmodes or constrained degrees of freedom can be performed, we must first transform the equations to a new space (with redefined degrees of freedom) in such a way that the degrees of freedom to be eliminated are clearly identified. Thus we need to choose exactly which of the constrained degrees of freedom (in each constraint equation) and which degrees of freedom related to the translational and rotational modes are to be eliminated. In our implementation, we choose to eliminate the translations and rotation of the first node, and we implement a strategic routine which chooses which of the other translational degrees of freedom related to the constraint equations are to be eliminated. The identification of the constrained degrees of freedom to be eliminated is performed by a transformation matrix $\mathbf{T}_{\mathrm{w}}^{c}$ in which each column belongs to a constraint equation and identifies the degree of freedom to be eliminated by a unit value in the corresponding row. The remaining degrees of freedom, which are not going to be separately identified (eliminated), are identified in the transformation matrix $\mathbf{T}_{\mathrm{w}}^{u}$ in which each column identifies a remaining ( $u$ for unconstrained) degree of freedom by a unit value in the corresponding row.

We are now ready to introduce the transformation to $\mathbf{v}_{\mathrm{w}}$-space from the new space. In the new space, we introduce the degrees of freedom as, $\mathbf{v}_{\mathrm{w}}^{\alpha}=\left[\begin{array}{ll}v_{\mathrm{w}}^{1} & v_{\mathrm{w}}^{2}\end{array}\right]^{T}$ for the magnitudes of the two translational eigenmodes, $v_{\mathrm{w}}^{3}$ for the magnitude of the rotational eigenmode, $\mathbf{v}_{\mathrm{w}}^{c}$ for the degrees of freedom to be constrained, and $\mathbf{v}_{\mathrm{w}}^{u}$ for the remaining unconstrained degrees of freedom. The transformation may be written as:

$\mathbf{v}_{\mathrm{w}}=\left[\begin{array}{llll}\mathbf{T}_{\mathrm{w}}^{\alpha} & \mathbf{T}_{\mathrm{w}}^{3} & \mathbf{T}_{\mathrm{w}}^{c} & \mathbf{T}_{\mathrm{w}}^{u}\end{array}\right]\left[\begin{array}{c}\mathbf{v}_{\mathrm{w}}^{\alpha} \\ v_{\mathrm{w}}^{3} \\ \mathbf{v}_{\mathrm{w}}^{c} \\ \mathbf{v}_{\mathrm{w}}^{u}\end{array}\right]$

Since we have strategically chosen the constrained degrees of freedom not to be equal to the degrees of freedom related to the translational and rotational eigenmodes, we have a situation where $\mathbf{C T}_{\mathrm{w}}^{\alpha}=\mathbf{0}$ and $\mathbf{C} \mathbf{T}_{\mathrm{w}}^{3}=$ 0 . So the constrain equations in (42) can be rewritten using equation (43) as

$\mathbf{C} \mathbf{T}_{\mathrm{w}}^{c} \mathbf{v}_{\mathrm{w}}^{c}+\mathbf{C} \mathbf{T}_{\mathrm{w}}^{u} \mathbf{v}_{\mathrm{w}}^{u}=\mathbf{0}$ 
This allows us to express the constrained degrees of freedom by the unconstrained as

$\mathbf{v}_{\mathrm{w}}^{c}=-\mathbf{C}_{c}^{-1} \mathbf{C}_{u} \mathbf{v}_{\mathrm{w}}^{u}$

in which $\mathbf{C}_{c}=\mathbf{C} \mathbf{T}_{\mathrm{w}}^{c}$ and $\mathbf{C}_{u}=\mathbf{C T}_{\mathrm{w}}^{u}$. Introducing (45) in the transformation equation (43), we find that the total transformation is condensed as follows:

$$
\begin{aligned}
\mathbf{v}_{\mathrm{w}} & =\left[\begin{array}{llll}
\mathbf{T}_{\mathrm{w}}^{\alpha} & \mathbf{T}_{\mathrm{w}}^{3} & \mathbf{T}_{\mathrm{w}}^{c} & \mathbf{T}_{\mathrm{w}}^{u}
\end{array}\right]\left[\begin{array}{c}
\mathbf{v}_{\mathrm{w}}^{\alpha} \\
v_{\mathrm{w}}^{3} \\
-\mathbf{C}_{c}{ }^{-1} \mathbf{C}_{u} \mathbf{v}_{\mathrm{w}}^{u} \\
\mathbf{v}_{\mathrm{w}}^{u}
\end{array}\right] \\
& =\left[\begin{array}{lll}
\mathbf{T}_{\mathrm{w}}^{\alpha} & \mathbf{T}_{\mathrm{w}}^{3} & \widetilde{\mathbf{T}}_{\mathrm{w}}^{u}
\end{array}\right]\left[\begin{array}{c}
\mathbf{v}_{\mathrm{w}}^{\alpha} \\
v_{\mathrm{w}}^{3} \\
\mathbf{v}_{\mathrm{w}}^{u}
\end{array}\right]
\end{aligned}
$$

where $\widetilde{\mathbf{T}}_{\mathrm{w}}^{u}=\mathbf{T}_{\mathrm{w}}^{u}-\mathbf{T}_{\mathrm{w}}^{c} \mathbf{C}_{c}{ }^{-1} \mathbf{C}_{u}$ has been introduced as the condensed transformation by using a tilde.

Introducing the transformation in (46) in the differential equations in (33) transforms these equations into the new space. The differential equations thereby take the following form in which we have also introduced the null terms corresponding to the rigid-body modes and zero shear strain for translational and flexural modes:

$$
\begin{gathered}
{\left[\begin{array}{ccc}
\mathbf{K}_{\alpha \alpha}^{\sigma} & \mathbf{0} & \mathbf{K}_{\alpha u}^{\sigma} \\
\mathbf{0} & K_{33}^{\sigma} & \mathbf{K}_{3 u}^{\sigma} \\
\mathbf{K}_{u \alpha}^{\sigma} & \mathbf{K}_{u 3}^{\sigma} & \mathbf{K}_{u u}^{\sigma}
\end{array}\right]\left[\begin{array}{c}
\mathbf{v}_{\mathrm{w}}^{\alpha} \\
v_{\mathrm{w}}^{3} \\
\mathbf{v}_{\mathrm{w}}^{\mathbf{u}}
\end{array}\right] \psi^{\prime \prime \prime \prime}-\left[\begin{array}{ccc}
\mathbf{0} & \mathbf{0} & \mathbf{0} \\
\mathbf{0} & K_{33}^{\tau} & \mathbf{K}_{3 u}^{\tau} \\
\mathbf{0} & \mathbf{K}_{u 3}^{\tau} & \mathbf{K}_{u u}^{\tau}
\end{array}\right]\left[\begin{array}{c}
\mathbf{v}_{\mathrm{w}}^{\alpha} \\
v_{\mathrm{w}}^{3} \\
\mathbf{v}_{\mathrm{w}}^{\mathbf{u}}
\end{array}\right] \psi^{\prime \prime}} \\
+\left[\begin{array}{ccc}
\mathbf{0} & \mathbf{0} & \mathbf{0} \\
\mathbf{0} & 0 & \mathbf{0} \\
\mathbf{0} & \mathbf{0} & \mathbf{K}_{u u}^{s}
\end{array}\right]\left[\begin{array}{c}
\mathbf{v}_{\mathrm{w}}^{\alpha} \\
v_{\mathrm{w}}^{3} \\
\mathbf{v}_{\mathrm{w}}^{\mathbf{u}}
\end{array}\right] \psi=\left[\begin{array}{l}
\mathbf{0} \\
0 \\
\mathbf{0}
\end{array}\right]
\end{gathered}
$$

The transformed stiffness matrices introduced in this equation are given in Table 4. The two-dimensional upper block matrix equation yields the translation displacements as

$\mathbf{v}_{\mathrm{w}}^{\alpha} \psi^{\prime \prime \prime \prime}=-\mathbf{K}_{\alpha \alpha}^{\sigma-1} \mathbf{K}_{\alpha u}^{\sigma} \mathbf{v}_{\mathrm{w}}^{u} \psi^{\prime \prime \prime \prime}$

We can identify the two orthogonal pure translational modes, $\left(v_{\mathrm{w}}^{1}, v_{\mathrm{w}}^{2}, v_{\mathrm{w}}^{3}, \mathbf{v}_{\mathrm{w}}^{u}\right)=(1,0,0, \mathbf{0})$, and,$(0,1,0, \mathbf{0})$, as eigenmodes or solutions to equation (47). For these pure translational modes, we find that the right-hand side of equation (48) vanishes and that the axial variation of the pure translational modes is therefore determined by quadruple integration, which gives:

$$
\begin{aligned}
& \psi_{1}(z)=c_{11}+c_{12} z+c_{13} z^{2}+c_{14} z^{3}=\Psi_{1}(z) \mathbf{c}_{1} \\
& \psi_{2}(z)=c_{21}+c_{22} z+c_{23} z^{2}+c_{24} z^{3}=\Psi_{2}(z) \mathbf{c}_{2}
\end{aligned}
$$

We will make use of the following block notation:

$$
\boldsymbol{\Psi}_{\alpha} \mathbf{c}_{\alpha}=\left[\begin{array}{cc}
\boldsymbol{\Psi}_{1} & \mathbf{0} \\
\mathbf{0} & \boldsymbol{\Psi}_{2}
\end{array}\right]\left[\begin{array}{l}
\mathbf{c}_{1} \\
\mathbf{c}_{2}
\end{array}\right], \mathbf{c}_{1}=\left[\begin{array}{l}
c_{11} \\
c_{12} \\
c_{13} \\
c_{14}
\end{array}\right], \mathbf{c}_{2}=\left[\begin{array}{l}
c_{21} \\
c_{22} \\
c_{23} \\
c_{24}
\end{array}\right]
$$

in which $\boldsymbol{\Psi}_{1}=\boldsymbol{\Psi}_{2}=\left[\begin{array}{llll}1 & z & z^{2} & z^{3}\end{array}\right]$. The constants in the vectors $\mathbf{c}_{1}$ and $\mathbf{c}_{2}$ are determined by the boundary conditions for pure transverse translational displacement in the two directions.

Having identified the two pure translational modes, we turn to the remaining solutions to the differential equation (47). In this case, equation (48) determines the correction term that eliminates pure transverse displacements in the back substitution process. By dividing both sides of the equation by $\psi^{\prime \prime \prime \prime} \neq 0$ we find

$\mathbf{v}_{\mathrm{w}}^{\alpha}=-\mathbf{K}_{\alpha \alpha}^{\sigma-1} \mathbf{K}_{\alpha u}^{\sigma} \mathbf{v}_{\mathrm{w}}^{u}$

Using this equation or equation (48), we eliminate the two pure flexural degrees of freedom and find the condensed version of the differential equation (47), which takes the following form:

$$
\begin{gathered}
{\left[\begin{array}{cc}
K_{33}^{\sigma} & \mathbf{K}_{3 u}^{\sigma} \\
\mathbf{K}_{u 3}^{\sigma} & \overline{\mathbf{K}}_{u u}^{\sigma}
\end{array}\right]\left[\begin{array}{c}
v_{\mathrm{w}}^{3} \\
\mathbf{v}_{\mathrm{w}}^{u}
\end{array}\right] \psi^{\prime \prime \prime \prime}-\left[\begin{array}{ll}
K_{33}^{\tau} & \mathbf{K}_{3 u}^{\tau} \\
\mathbf{K}_{u 3}^{\tau} & \mathbf{K}_{u u}^{\tau}
\end{array}\right]\left[\begin{array}{c}
v_{\mathrm{w}}^{3} \\
\mathbf{v}_{\mathrm{w}}^{u}
\end{array}\right] \psi^{\prime \prime}} \\
+\left[\begin{array}{cc}
0 & \mathbf{0} \\
\mathbf{0} & \mathbf{K}_{u u}^{s}
\end{array}\right]\left[\begin{array}{l}
v_{\mathrm{w}}^{3} \\
\mathbf{v}_{\mathrm{w}}^{u}
\end{array}\right] \psi=\left[\begin{array}{l}
0 \\
\mathbf{0}
\end{array}\right]
\end{gathered}
$$

The introduced stiffness matrix $\overline{\mathbf{K}}_{u u}^{\sigma}$ is given in Table 4. This equation constitutes the GBT differential equations constrained by shear flow constraints and wallwidth constraints after the elimination of the classical axial and two translational (flexural beam) modes.

\subsection{Step III - Reduction of order and pure St. Venant torsion}

In the following, we will reduce the differential order of the coupled fourth order differential equations and the related quadratic eigenvalue problem to twice as many coupled second order differential equations with a related linear eigenvalue problem of double size. This method is equivalent to the one used for the solution of the coupled homogeneous problem of one-mode distortion and torsion analyzed in [12]. After we have changed the order of the equations, we can recognize that the pure torsional St. Venant displacement modes with a constant or a linear variation of the angle of twist are eigensolutions.

The fourth order differential equation (52) can be transformed into twice as many second order differential equations by introducing what is called a state vector. There are a number of different possible formulations, but we have chosen the use of the state vector $\mathbf{v}_{S}=\left[v_{\mathrm{w}}^{3} \psi, \mathbf{v}_{\mathrm{w}}^{u} \psi, v_{\mathrm{w}}^{3} \psi^{\prime \prime}, \mathbf{v}_{\mathrm{w}}^{u} \psi^{\prime \prime}\right]^{T}$. Introducing this state vector (and using related equality block equations) yields a reformulation of equation (52) as a formal second order matrix differential equation of double size, 


\begin{tabular}{lcl}
\hline $\mathbf{K}_{\alpha \alpha}^{\sigma}=\mathbf{T}_{\mathrm{w}}^{\alpha T} \mathbf{K}^{\sigma} \mathbf{T}_{\mathrm{w}}^{\alpha}$ & $K_{33}^{\sigma}=\mathbf{T}_{\mathrm{w}}^{3 T} \mathbf{K}^{\sigma} \mathbf{T}_{\mathrm{w}}^{3}$ & $K_{33}^{\tau}=\mathbf{T}_{\mathrm{w}}^{3 T} \mathbf{K}^{\tau} \mathbf{T}_{\mathrm{w}}^{3}$ \\
$\mathbf{K}_{\alpha z}^{\sigma}=\mathbf{T}_{\mathrm{w}}^{\alpha T} \mathbf{K}^{\sigma} \mathbf{T}_{\mathrm{w}}^{\mathbf{z}}$ & $\mathbf{K}_{3 u}^{\sigma}=\mathbf{T}_{\mathrm{w}}^{3} \mathbf{K}^{\sigma} \widetilde{\mathbf{T}}_{\mathrm{w}}^{\mathrm{u}}$ & $\mathbf{K}_{3 u}^{\tau}=\mathbf{T}_{\mathrm{w}}^{3} \mathbf{K}^{\tau} \widetilde{\mathbf{T}}_{\mathrm{w}}^{\mathrm{u}}$ \\
$\mathbf{K}_{\alpha u}^{\sigma}=\mathbf{T}_{\mathrm{w}}^{\alpha T} \mathbf{K}^{\sigma} \widetilde{\mathbf{T}}_{\mathrm{w}}^{\mathrm{u}}$ & $\mathbf{K}_{u u}^{\sigma}=\widetilde{\mathbf{T}}_{\mathrm{w}}^{u T} \mathbf{K}^{\sigma} \widetilde{\mathbf{T}}_{\mathrm{w}}^{\mathrm{u}}$ & $\mathbf{K}_{u u}^{\tau}=\widetilde{\mathbf{T}}_{\mathrm{w}}^{u T} \mathbf{K}^{\tau} \widetilde{\mathbf{T}}_{\mathrm{w}}^{\mathbf{u}}$ \\
$\mathbf{K}_{u u}^{s}=\widetilde{\mathbf{T}}_{\mathrm{w}}^{u} \mathbf{K}^{s} \widetilde{\mathbf{T}}_{\mathrm{w}}^{u}$ & $\overline{\mathbf{K}}_{u u}^{\sigma}=\mathbf{K}_{u u}^{\sigma}-\mathbf{K}_{u \alpha}^{\sigma} \mathbf{K}_{\alpha \alpha}^{\sigma}{ }^{-1} \mathbf{K}_{\alpha u}^{\sigma}$ & \\
\hline
\end{tabular}

Table 4: Transformation of stiffness matrices related to Step II.

which takes the form:

$$
\begin{gathered}
{\left[\begin{array}{cccc}
0 & \mathbf{0} & 0 & \mathbf{0} \\
\mathbf{0} & \mathbf{K}_{u u}^{s} & \mathbf{0} & \mathbf{0} \\
0 & \mathbf{0} & -K_{33}^{\sigma} & -\mathbf{K}_{3 u}^{\sigma} \\
\mathbf{0} & \mathbf{0} & -\mathbf{K}_{u 3}^{\sigma} & -\overline{\mathbf{K}}_{u u}^{\sigma}
\end{array}\right]\left[\begin{array}{c}
v_{\mathrm{w}}^{3} \psi \\
\mathbf{v}_{\mathrm{w}}^{u} \psi \\
v_{\mathrm{w}}^{3} \psi^{\prime \prime} \\
\mathbf{v}_{\mathrm{w}}^{u} \psi^{\prime \prime}
\end{array}\right]} \\
-\left[\begin{array}{cccc}
K_{33}^{\tau} & \mathbf{K}_{3 u}^{\tau} & -K_{33}^{\sigma} & -\mathbf{K}_{3 u}^{\sigma} \\
\mathbf{K}_{u 3}^{\tau} & \mathbf{K}_{u u}^{\tau} & -\mathbf{K}_{u 3}^{\sigma} & -\overline{\mathbf{K}}_{u u}^{\sigma} \\
-K_{33}^{\sigma} & -\mathbf{K}_{3 u}^{\sigma} & 0 & \mathbf{0} \\
-\mathbf{K}_{u 3}^{\sigma} & -\overline{\mathbf{K}}_{u u}^{\sigma} & \mathbf{0} & \mathbf{0}
\end{array}\right]\left[\begin{array}{c}
v_{\mathrm{w}}^{3} \psi \\
\mathbf{v}_{\mathrm{w}}^{u} \psi \\
v_{\mathrm{w}}^{3} \psi^{\prime \prime} \\
\mathbf{v}_{\mathrm{w}}^{u} \psi^{\prime \prime}
\end{array}\right]=\left[\begin{array}{l}
0 \\
\mathbf{0} \\
0 \\
\mathbf{0}
\end{array}\right]
\end{gathered}
$$

Seeking solutions of exponential form, $\psi(z)=e^{\xi z}$, with an eigenvector in which $v_{\mathrm{w}}^{3}=1$ and $\mathbf{v}_{w}^{u}=\mathbf{0}$, we see that the first equation will lead to an eigenvalue, $\xi^{2}=0$, or a double zero root (in the characteristic equation), thus giving us not exponential solutions but two linear solution terms. This corresponds to a constant or a linear variation of the first degree of freedom, which is pure twist. However if we "persistently" seek the two classical exponential solutions for a pure twist mode with (eigen)vectors, $\left(1, \mathbf{0}, \xi^{2}, \mathbf{0}\right)^{T}$, we are not able to show that this is in general a solution. In the examples section, we will see that for the closed cross-section, only the linear terms of pure twist exist, whereas for the open channel section, the eigenvalue is very close to the classical result, and in the example chosen, we cannot visually see the distortion in the associated "torsional" mode with an exponential variation of twist.

To keep the matrix operations as simple as possible we introduce a new vector $\mathbf{v}_{\mathrm{w}}^{e}$ and three new block matrices, $\mathbf{K}_{e e}^{\sigma}, \mathbf{K}_{3 e}^{\sigma}$ and $\mathbf{K}_{u e}^{\sigma}$, given by

$$
\left.\mathbf{v}_{\mathrm{w}}^{e}=\left[\begin{array}{c}
v_{\mathrm{w}}^{3} \\
\mathbf{v}_{\mathrm{w}}^{u}
\end{array}\right] \quad \mathbf{K}_{e e}^{\sigma}=\left[\begin{array}{c}
\mathbf{K}_{3 e}^{\sigma} \\
\mathbf{K}_{u e}^{\sigma}
\end{array}\right]=\left[\begin{array}{cc}
{\left[K_{33}^{\sigma}\right.} & \mathbf{K}_{3 u}^{\sigma} \\
{\left[\mathbf{K}_{u 3}^{\sigma}\right.} & \overline{\mathbf{K}}_{u u}^{\sigma}
\end{array}\right]\right]
$$

When we introduce the new vector and the three block matrices defined by equation (54) and in Table 5, the second order differential equations can be written as:

$$
\begin{aligned}
& {\left[\begin{array}{ccc}
0 & \mathbf{0} & \mathbf{0} \\
\mathbf{0} & \mathbf{K}_{u u}^{s} & \mathbf{0} \\
\mathbf{0} & \mathbf{0} & -\mathbf{K}_{e e}^{\sigma}
\end{array}\right]\left[\begin{array}{c}
v_{\mathrm{w}}^{3} \psi \\
\mathbf{v}_{\mathrm{w}}^{u} \psi \\
\mathbf{v}_{\mathrm{w}}^{e} \psi^{\prime \prime}
\end{array}\right]} \\
& -\left[\begin{array}{ccc}
K_{33}^{\tau} & \mathbf{K}_{3 u}^{\tau} & -\mathbf{K}_{3 e}^{\sigma} \\
\mathbf{K}_{u 3}^{\tau} & \mathbf{K}_{u u}^{\tau} & -\mathbf{K}_{u e}^{\sigma} \\
-\mathbf{K}_{e 3}^{\sigma} & -\mathbf{K}_{e u}^{\sigma} & \mathbf{0}
\end{array}\right]\left[\begin{array}{c}
v_{\mathrm{w}}^{3} \psi \\
\mathbf{v}_{\mathbf{w}}^{u} \psi \\
\mathbf{v}_{\mathrm{w}}^{e} \psi^{\prime \prime}
\end{array}\right]^{\prime \prime}=\left[\begin{array}{l}
0 \\
\mathbf{0} \\
\mathbf{0}
\end{array}\right]
\end{aligned}
$$

In the first equation we can isolate the pure rotational term resulting in the following differential equation:

$v_{\mathrm{w}}^{3} \psi^{\prime \prime}=-K_{33}^{\tau}{ }^{-1}\left(\mathbf{K}_{3 u}^{\tau} \mathbf{v}_{\mathrm{w}}^{u} \psi^{\prime \prime}-\mathbf{K}_{3 e}^{\sigma} \mathbf{v}_{\mathrm{w}}^{\mathbf{e}} \psi^{\prime \prime \prime \prime}\right)$

It can be seen that pure St. Venant torsion (with free warping), corresponding to the solution vector, $\left(v_{\mathrm{w}}^{3} \psi, \mathbf{v}_{\mathrm{w}}^{u} \psi, \mathbf{v}_{\mathrm{w}}^{e} \psi^{\prime \prime}\right)=\left(c_{32} z+c_{31}, \mathbf{0}, \mathbf{0}\right)$, is a solution of the second order differential equations in (55). We have thus shown that

$$
\begin{aligned}
\psi_{3}(z) & =c_{31}+c_{32} z \\
& =\Psi_{3}(z) \mathbf{c}_{3}=\left[\begin{array}{ll}
1 & z
\end{array}\right]\left[\begin{array}{l}
c_{31} \\
c_{32}
\end{array}\right]
\end{aligned}
$$

The remaining solutions to the differential equations in (55) are found by seeking exponential solutions of the form $\psi(z)=e^{\xi z}$. We insert the exponential solution in equation (56) and find the following equation, which we will use for back-substitution purposes:

$v_{\mathrm{w}}^{3}=-K_{33}^{\tau}{ }^{-1}\left(\mathbf{K}_{3 u}^{\tau} \mathbf{v}_{\mathrm{w}}^{u}-\mathbf{K}_{3 e}^{\sigma}\left(\xi^{2} \mathbf{v}_{\mathrm{w}}^{\mathbf{e}}\right)\right)$

Using equation (56), we eliminate $v_{\mathrm{w}}^{3}$ from the differential equations in (55) and find the final distortional differential equations of GBT that determine all the distortional displacement modes as

$$
\left[\begin{array}{cc}
\mathbf{K}_{u u}^{s} & \mathbf{0} \\
\mathbf{0} & -\mathbf{K}_{e e}^{\sigma}
\end{array}\right]\left[\begin{array}{c}
\mathbf{v}_{\mathrm{w}}^{u} \psi \\
\mathbf{v}_{\mathrm{w}}^{e} \psi^{\prime \prime}
\end{array}\right]-\left[\begin{array}{cc}
\overline{\mathbf{K}}_{u u}^{\tau} & -\overline{\mathbf{K}}_{u e}^{\sigma} \\
-\overline{\mathbf{K}}_{e u}^{\sigma} & -\overline{\mathbf{K}}_{e e}^{\sigma}
\end{array}\right]\left[\begin{array}{c}
\mathbf{v}_{\mathrm{w}}^{u} \psi \\
\mathbf{v}_{\mathrm{w}}^{e} \psi^{\prime \prime}
\end{array}\right]^{\prime \prime}=\left[\begin{array}{l}
\mathbf{0} \\
\mathbf{0}
\end{array}\right]
$$

The block matrices and the transformed stiffness matrices are given in Table 5. In the following section we will describe the solution of these differential equations. 


\begin{tabular}{lll}
$\mathbf{K}_{3 e}^{\sigma}=\left[\begin{array}{lll}K_{33}^{\sigma} & \mathbf{K}_{3 u}^{\sigma}\end{array}\right]$ & $\mathbf{K}_{u e}^{\sigma}=\left[\begin{array}{ll}\mathbf{K}_{u 3}^{\sigma} & \overline{\mathbf{K}}_{u u}^{\sigma}\end{array}\right]$ & $\mathbf{K}_{e e}^{\sigma T}=\left[\begin{array}{ll}\mathbf{K}_{3 e}^{\sigma T} & \mathbf{K}_{u e}^{\sigma T}\end{array}\right]$ \\
$\overline{\mathbf{K}}_{e e}^{\sigma}=\mathbf{K}_{e 3}^{\sigma} K_{33}^{\tau}{ }^{-1} \mathbf{K}_{3 e}^{\sigma}$ & $\overline{\mathbf{K}}_{u e}^{\sigma}=\mathbf{K}_{u e}^{\sigma}-\mathbf{K}_{u 3}^{\tau} K_{33}^{\tau}{ }^{-1} \mathbf{K}_{3 e}^{\sigma}$ & $\overline{\mathbf{K}}_{u u}^{\tau}=\mathbf{K}_{u u}^{\tau}-\mathbf{K}_{u 3}^{\tau} K_{33}^{\tau}{ }^{-1} \mathbf{K}_{3 u}^{\tau}$ \\
\hline
\end{tabular}

Table 5: Transformation of stiffness matrices related to Step III.

\section{The distortional eigenvalue problem and homoge- neous solution functions}

To find the distortional eigenmodes, including what are called local modes, we are finally able to seek solutions to the final condensed differential matrix equation (59). We postulate that the solutions are exponential solutions of the form

$\psi(z)=e^{\xi z}$

where $\xi$ is an inverse length scale parameter which may be complex. Inserting the postulated solution leads to the following generalized linear matrix eigenvalue problem, in which the eigenvalues are $\xi^{2}$ and the eigenvectors are the distortional modes that we seek:

$\left[\begin{array}{cc}\mathbf{K}_{u u}^{s} & \mathbf{0} \\ \mathbf{0} & -\mathbf{K}_{e e}^{\sigma}\end{array}\right]\left[\begin{array}{c}\mathbf{v}_{\mathbf{w}}^{u} \\ \xi^{2} \mathbf{v}_{\mathrm{w}}^{e}\end{array}\right]-\xi^{2}\left[\begin{array}{cc}\overline{\mathbf{K}}_{u u}^{\tau} & -\overline{\mathbf{K}}_{u e}^{\sigma} \\ -\overline{\mathbf{K}}_{e u}^{\sigma} & -\overline{\mathbf{K}}_{e e}^{\sigma}\end{array}\right]\left[\begin{array}{c}\mathbf{v}_{\mathrm{w}}^{u} \\ \xi^{2} \mathbf{v}_{\mathrm{w}}^{e}\end{array}\right]=\left[\begin{array}{l}\mathbf{0} \\ \mathbf{0}\end{array}\right]$

Due to the differences in the order of magnitude of the different stiffness terms in the matrices, we have chosen to improve the numerical accuracy of the eigenvalue and eigenvector solution in our numerical implementation by introducing the following Cholesky decomposition of the block matrices in the first matrix:

$$
\mathbf{K}_{u u}^{s}=\mathbf{L}_{u} \mathbf{L}_{u}^{T} \quad \mathbf{K}_{e e}^{\sigma}=\mathbf{L}_{e} \mathbf{L}_{e}^{T}
$$

We utilize the decomposition by introducing the following new intermediate vectors

$$
\mathbf{v}_{\mathrm{w}}^{u}=\mathbf{L}_{u}^{-T} \widetilde{\mathbf{v}}_{\mathrm{w}}^{u} \quad\left(\xi^{2} \mathbf{v}_{\mathrm{w}}^{e}\right)=\mathbf{L}_{e}^{-T}\left(\xi^{2} \widetilde{\mathbf{v}}_{\mathrm{w}}^{e}\right)
$$

where the superscript ${ }^{-T}$ corresponds to the inverted transpose of the matrix. After pre-multiplication of each block matrix equation by $\mathbf{L}_{u}^{-1}$ and $\mathbf{L}_{e}^{-1}$, the eigenvalue problem then takes the following form:

$$
\begin{aligned}
& {\left[\begin{array}{cc}
\mathbf{I}_{u u} & \mathbf{0} \\
\mathbf{0} & -\mathbf{I}_{e e}
\end{array}\right]\left[\begin{array}{c}
\widetilde{\mathbf{v}}_{\mathrm{w}}^{u} \\
\xi^{2} \widetilde{\mathbf{v}}_{\mathrm{w}}^{e}
\end{array}\right]} \\
& \quad-\xi^{2}\left[\begin{array}{cc}
\mathbf{L}_{u}^{-1} \overline{\mathbf{K}}_{u u}^{\tau} \mathbf{L}_{u}^{-T} & -\mathbf{L}_{u}^{-1} \overline{\mathbf{K}}_{u e}^{\sigma} \mathbf{L}_{e}^{-T} \\
-\mathbf{L}_{e}^{-1} \overline{\mathbf{K}}_{e u}^{\sigma} \mathbf{L}_{u}^{-T} & -\mathbf{L}_{e}^{-1} \overline{\mathbf{K}}_{e e}^{\sigma} \mathbf{L}_{e}^{-T}
\end{array}\right]\left[\begin{array}{c}
\widetilde{\mathbf{v}}_{\mathrm{w}}^{u} \\
\xi^{2} \widetilde{\mathbf{v}}_{\mathrm{w}}^{e}
\end{array}\right]=\left[\begin{array}{c}
\mathbf{0} \\
\mathbf{0}
\end{array}\right]
\end{aligned}
$$

In this equation $\mathbf{I}_{u u}$ and $\mathbf{I}_{e e}$ are adequate size unit diagonal matrices. Some general eigenvalue solution routines demand that at least one of the matrices has to be symmetric as well as positive (semi-)definite. This can be achieved by a change of sign in the second block matrix equation, however the second matrix becomes asymmetric. Having found the eigenvectors, we use equation (63) to find $\mathbf{v}_{\mathrm{w}}^{u}$ and $\left(\xi^{2} \mathbf{v}_{\mathrm{w}}^{u}\right)$, which can then be used for the remaining back-transformation process.

Each distortional eigenvector corresponds to a solution $\psi_{d_{i}}(z)$ of the homogeneous coupled equations of distortion in equation (59). The solution function corresponds to our trial function in equation (60), and it has now been determined by plus/minus the square root of the eigenvalues as $\pm \xi_{i}$. In other words, for the $i$ 'th eigenvector we find the solution

$$
\begin{aligned}
\psi_{d_{i}}(z) & =c_{d_{2 i-1}} e^{\xi_{i} z}+c_{d_{2 i}} e^{-\xi_{i} z} \\
& =\Psi_{d_{i}}(z) \mathbf{c}_{d_{i}}=\left[\begin{array}{ll}
e^{\xi_{i} z} & e^{-\xi_{i} z}
\end{array}\right]\left[\begin{array}{c}
c_{d_{2 i-1}} \\
c_{d_{2 i}}
\end{array}\right]
\end{aligned}
$$

in which constants $c_{d_{2 i-1}}$ and $c_{d_{2 i}}$ assembled in the vector $\mathbf{c}_{d_{i}}$ depend on the boundary conditions of the problem at hand. All the distortional solution functions are assembled in the distortional solution matrix $\Psi_{d}$ and multiplied by the assembled vector of distortional constants $\mathbf{c}_{d}$ as follows:

$\boldsymbol{\Psi}_{d}(z) \mathbf{c}_{d}=\left[\begin{array}{cccc}\boldsymbol{\Psi}_{d_{1}}(z) & \mathbf{0} & \mathbf{0} & \cdots \\ \mathbf{0} & \boldsymbol{\Psi}_{d_{2}}(z) & \mathbf{0} & \ldots \\ \mathbf{0} & \mathbf{0} & \boldsymbol{\Psi}_{d_{3}}(z) & \cdots \\ \vdots & \vdots & \vdots & \ddots\end{array}\right]\left[\begin{array}{c}\mathbf{c}_{d_{1}} \\ \mathbf{c}_{d_{2}} \\ \mathbf{c}_{d_{3}} \\ \vdots\end{array}\right]$

This notation is used later to describe the total displacement solution.

\section{Back substitution}

Having found the distortional eigenvalues, eigenvectors and homogeneous solutions for the reduced system (61), we now have to perform a backward substitution through the previous steps in order to achieve the results in the original displacement vector format. Furthermore, we also have to back-substitute all eliminated eigenvectors (multiple zero eigenvalues) and review the related homogeneous solutions.

\subsection{Back substitution of distortional modes}

In the previous sections, the formulations are related to a single displacement vector and the back substitution 
of the distortional modes found from solving the eigenvalue problem in equation (61) is performed sequentially through equations (58), (51), (46), (32) and (24). In a typical modal approach, all eigenvectors are assembled column-wise in the mode matrix and the related eigenvalues $\xi^{2}$ are placed sequentially in the diagonal of the matrix $\boldsymbol{\Lambda}$. By introducing the capital letter $\mathbf{V}$ with related sub- and superscripts for the assembled modes, we can find the back-substituted distortional mode matrices $\mathbf{V}_{\mathrm{w}}^{d}$ and $\mathbf{V}_{\Omega}^{d}$ using the following sequence of substitutions corresponding to the sequence of equations mentioned above:

$$
\begin{aligned}
\mathbf{V}_{\mathrm{w}}^{3} & =-K_{33}^{\tau-1}\left(\mathbf{K}_{3 u}^{\tau} \mathbf{V}_{\mathrm{w}}^{u}-\mathbf{K}_{3 e}^{\sigma}\left(\mathbf{V}_{\mathrm{w}}^{\mathrm{e}} \mathbf{\Lambda}\right)\right) \\
\mathbf{V}_{\mathrm{w}}^{\alpha} & =-\mathbf{K}_{\alpha \alpha}^{\sigma}{ }^{-1} \mathbf{K}_{\alpha u}^{\sigma} \mathbf{V}_{\mathrm{w}}^{u} \\
\mathbf{V}_{\mathrm{w}}^{d} & =\mathbf{T}_{\mathrm{w}}^{\alpha} \mathbf{V}_{\mathrm{w}}^{\alpha}+\mathbf{T}_{\mathrm{w}}^{3} \mathbf{V}_{\mathrm{w}}^{3}+\widetilde{\mathbf{T}}_{\mathrm{w}}^{u} \mathbf{V}_{\mathrm{w}}^{u} \\
\mathbf{V}_{\Omega}^{d, a} & =-\left(K_{\Omega \Omega}^{\sigma a a}\right)^{-1} \mathbf{K}_{\Omega \Omega}^{\sigma a r} \mathbf{V}_{\mathrm{w}}^{d} \\
\mathbf{V}_{\Omega}^{d} & =\mathbf{T}_{\Omega \mathrm{w}}^{r} \mathbf{V}_{\mathrm{w}}^{d}+\mathbf{T}_{\Omega}^{a} \mathbf{V}_{\Omega}^{d, a}
\end{aligned}
$$

The superscript $d$ has been introduced to distinguish the distortional modes from the total assembly of modes introduced later. The term $\left(\mathbf{V}_{\mathrm{w}}^{\mathrm{e}} \mathbf{\Lambda}\right)$ is just one matrix, which is never separated into the two product terms, but just found directly as part of the eigenvectors of the reduced-order eigenvalue problem in equation (61).

\subsection{Back substitution of eliminated beam displacement modes}

The back substitution of eliminated beam displacement modes involves back substitution of the pure axial extension mode, the two transverse translational modes, and the pure twist mode. Using the degree-of-freedom space introduced in Step II, these modes are given by the following four transverse displacement modal vectors:

$$
\left[\begin{array}{c}
v_{\mathrm{w}}^{1} \\
v_{\mathrm{w}}^{2} \\
v_{\mathrm{w}}^{3} \\
\mathbf{v}_{\mathrm{w}}^{u}
\end{array}\right]=\left[\begin{array}{c}
0 \\
0 \\
0 \\
\mathbf{0}
\end{array}\right],\left[\begin{array}{c}
v_{\mathrm{w}}^{1} \\
v_{\mathrm{w}}^{2} \\
v_{\mathrm{w}}^{3} \\
\mathbf{v}_{\mathrm{w}}^{u}
\end{array}\right]=\left[\begin{array}{c}
1 \\
0 \\
0 \\
\mathbf{0}
\end{array}\right],\left[\begin{array}{c}
v_{\mathrm{w}}^{1} \\
v_{\mathrm{w}}^{2} \\
v_{\mathrm{w}}^{3} \\
\mathbf{v}_{\mathrm{w}}^{u}
\end{array}\right]=\left[\begin{array}{c}
0 \\
1 \\
0 \\
\mathbf{0}
\end{array}\right],\left[\begin{array}{c}
v_{\mathrm{w}}^{1} \\
v_{\mathrm{w}}^{2} \\
v_{\mathrm{w}}^{3} \\
\mathbf{v}_{\mathrm{w}}^{u}
\end{array}\right]=\left[\begin{array}{c}
0 \\
0 \\
1 \\
\mathbf{0}
\end{array}\right]
$$

The first vector becomes the extensional eigenvector in the degree-of-freedom space introduced in Step I. The back substitution of these modes is all performed using the equation (46). However, we have already introduced the eigenvectors in the original transverse displacement space in the transformation matrices related to this equation, $\mathbf{T}_{\mathrm{w}}^{\alpha}$ and $\mathbf{T}_{\mathrm{w}}^{3}$ and the back transformation is obsolete for these modes. These eigenvectors are assembled in a beam mode matrix $\mathbf{V}_{\mathrm{w}}^{b}$ as follows:

$$
\mathbf{V}_{\mathrm{w}}^{b}=\left[\begin{array}{llll}
\mathbf{0} & \mathbf{v}_{\mathrm{w}}^{1} & \mathbf{v}_{\mathrm{w}}^{2 \operatorname{trans}} & \mathbf{v}_{\mathrm{w}}^{3 \text { rot }}
\end{array}\right]
$$

The back substitution of the warping displacements remains. Of course the pure axial extension warping vector is trivial and has allready been introduced as $\mathbf{T}_{\Omega}^{\text {axial }}=\mathbf{v}_{\Omega}^{\text {axial }}$, but we have to back-substitute the other modes. This is done by first calculating the axial (adjustment) component using equation (32) as follows:

$\mathbf{V}_{\Omega}^{b, a}=\left[\begin{array}{llll}1 & 0 & 0 & 0\end{array}\right]-\left(K_{\Omega \Omega}^{\sigma a a}\right)^{-1} \mathbf{K}_{\Omega \Omega}^{\sigma a r} \mathbf{V}_{\mathrm{w}}^{b}$

The beam warping vectors related to the transverse beam displacement modes can now be found using equation (24) as

$$
\begin{aligned}
& \mathbf{V}_{\Omega}^{b}=\left[\begin{array}{llll}
\mathbf{v}_{\Omega}^{\text {axial }} & \mathbf{v}_{\Omega}^{1 \text { trans }} & \mathbf{v}_{\Omega}^{2 \text { trans }} & \mathbf{v}_{\Omega}^{3} \text { rot }
\end{array}\right] \\
& =\mathbf{T}_{\Omega \mathrm{w}}^{r} \mathbf{V}_{\mathrm{W}}^{b}+\mathbf{T}_{\Omega}^{a} \mathbf{V}_{\Omega}^{b, a}
\end{aligned}
$$

The axial variation of the four modes has been identified in equations (31), (50) and (57) and can be assembled in the beam solution function matrix $\boldsymbol{\Psi}_{b}(z)$ and multiplied by the vector of beam displacement constants $\mathbf{c}_{b}$ as

$\boldsymbol{\Psi}_{b}(z) \mathbf{c}_{b}=\left[\begin{array}{ccc}\boldsymbol{\Psi}_{a}(z) & \mathbf{0} & \mathbf{0} \\ \mathbf{0} & \boldsymbol{\Psi}_{\alpha}(z) & \mathbf{0} \\ \mathbf{0} & \mathbf{0} & \boldsymbol{\Psi}_{3}(z)\end{array}\right]\left[\begin{array}{c}\mathbf{c}_{a} \\ \mathbf{c}_{\alpha} \\ \mathbf{c}_{3}\end{array}\right]$

in which we have introduced the integral of the axial solution as $\boldsymbol{\Psi}_{a}=\left[\begin{array}{ll}-z & -\frac{1}{2} z^{2}\end{array}\right]$. This matrix function does not pertain to any solution, and we might as well have set it to zero, since there are no transverse displacements, $\mathbf{v}_{\mathrm{w}}^{\text {axial }}=\mathbf{0}$, for the pure axial deformation mode in beam theory. However, this choice allows us to use the derivative of the $\boldsymbol{\Psi}_{a}(z)$ matrix, which is the axial solution $\boldsymbol{\Psi}_{a}^{\prime}=\left[\begin{array}{ll}-1 & -z\end{array}\right]$.

\section{The full homogenous solution}

The full homogenous solution can now be assembled from all the eigenmode vectors and the solution functions. It turns out that some of the eigenvalues and eigenvectors are complex. However in the following we will just perform a direct formulation in which we acknowledge that we are also dealing with complex quantities corresponding to related complex eigenvalues and complex axial solution functions. A transformation of the complex quantities to pairwise coupled real modes and real solution functions will be introduced in the second subsection.

\subsection{Direct formulation}

Let us assemble all the eigenvectors column-wise in a modal matrix of transverse displacement vectors $\mathbf{V}_{\mathrm{w}}$ and a modal matrix of axial warping displacement vectors 
$\mathbf{V}_{\Omega}$ by joining the modal matrices of the beam eigenvectors and the distortional eigenvectors in the following sequence

$$
\mathbf{V}_{\mathrm{w}}=\left[\begin{array}{ll}
\mathbf{V}_{\mathrm{w}}^{b} & \mathbf{V}_{\mathrm{w}}^{d}
\end{array}\right] \quad \mathbf{V}_{\Omega}=\left[\begin{array}{ll}
\mathbf{V}_{\Omega}^{b} & \mathbf{V}_{\Omega}^{d}
\end{array}\right]
$$

The solution function matrices and the displacement constant vectors can also be assembled using the previously defined block matrices and vectors

$$
\boldsymbol{\Psi}(z) \mathbf{c}=\left[\begin{array}{cc}
\boldsymbol{\Psi}_{b}(z) & \mathbf{0} \\
\mathbf{0} & \boldsymbol{\Psi}_{d}(z)
\end{array}\right]\left[\begin{array}{l}
\mathbf{c}_{b} \\
\mathbf{c}_{d}
\end{array}\right]
$$

The full homogeneous solution along the beam can be assembled in the nodal solution vectors $\mathbf{u}_{\mathrm{w}}(z)$ and $\mathbf{u}_{z}(z)$ as follows:

$$
\begin{aligned}
\mathbf{u}_{\mathrm{w}}(z) & =\mathbf{V}_{\mathrm{w}} \boldsymbol{\Psi}(z) \mathbf{c} \\
\mathbf{u}_{z}(z) & =-\mathbf{V}_{\Omega} \boldsymbol{\Psi}^{\prime}(z) \mathbf{c}
\end{aligned}
$$

The constants have to be determined by the boundary conditions of the thin-walled beam.

To be able to present and discuss unique complex eigenmodes, all the distortional vectors in $\mathbf{V}_{\mathrm{w}}$ have been normalized after back substitution in such a way that the largest absolute value of all components in each vector is one and that this component is real. This has to be done to make them unique since complex eigenvectors are determined except for an arbitrary complex constant.

\subsection{Transformation to real modes and real solution functions}

Some of the distortional displacement modes found and their related eigenvalues are complex. Mathematical formulation with the use of complex numbers simplify derivations and the use of compilers, which include complex numbers with complex operations on matrices, will lead to simple algorithms. However, it may be easier to grasp the form and meaning of the solution in real quantities.

Complex eigenvalues are always found as a pair of complex conjugated eigenvalues with eigenvectors which are also complex conjugated. For the $j$ 'th complex eigenvalue, $\xi_{j}^{2}$, and its conjugated $j+1$ 'th eigenvalue, $\xi_{j+1}^{2}=\overline{\xi_{j}^{2}}$, let us introduce the following notation for the positive square root values and the related eigenvector columns $\mathbf{v}_{j}$ and $\mathbf{v}_{j+1}$ of $\mathbf{V}_{\mathrm{w}}$ :

$$
\begin{array}{ll}
\xi_{j}=\lambda_{j}+\mu_{j} i & \xi_{j+1}=\bar{\xi}_{j}=\lambda_{j}-\mu_{j} i \\
\mathbf{v}_{j}=\mathbf{a}_{j}+\mathbf{b}_{j} i & \mathbf{v}_{j+1}=\overline{\mathbf{v}}_{j}=\mathbf{a}_{j}-\mathbf{b}_{j} i
\end{array}
$$

in which we have introduced the real and imaginary parts of the eigenvalues and eigenvectors. The complex eigenvectors in equation (81) may be conveniently written as

$\left[\begin{array}{ll}\mathbf{v}_{j} & \mathbf{v}_{j+1}\end{array}\right]=\left[\begin{array}{ll}\mathbf{a}_{j} & \mathbf{b}_{j}\end{array}\right]\left[\begin{array}{cc}1 & 1 \\ i & -i\end{array}\right]$

The constants of the related parts of the homogeneous solution are also complex quantities. However we are able to assemble the two complex conjugated modal solutions into two real (but pairwise coupled) solutions by introducing the real constant vectors $\widetilde{\mathbf{c}}_{j}$ and $\widetilde{\mathbf{c}}_{j+1}$ as follows:

$\left[\begin{array}{c}\mathbf{c}_{j} \\ \mathbf{c}_{j+1}\end{array}\right]=\frac{1}{2}\left[\begin{array}{cc}1 & -i \\ 1 & i\end{array}\right]\left[\begin{array}{c}\widetilde{\mathbf{c}}_{j} \\ \widetilde{\mathbf{c}}_{j+1}\end{array}\right]$

The $j$ 'th complex part of the full solution can now be rewritten using the transformations in equations (82) and (83). After multiplication and identification of real and imaginary parts, we find the following result:

$$
\begin{aligned}
& {\left[\begin{array}{ll}
\mathbf{v}_{j} & \mathbf{v}_{j+1}
\end{array}\right]\left[\begin{array}{cc}
\boldsymbol{\Psi}_{d_{j}} & \mathbf{0} \\
\mathbf{0} & \overline{\boldsymbol{\Psi}}_{d_{j}}
\end{array}\right]\left[\begin{array}{c}
\mathbf{c}_{j} \\
\mathbf{c}_{j+1}
\end{array}\right]} \\
& \quad=\left[\begin{array}{ll}
\mathbf{a}_{j} & \mathbf{b}_{j}
\end{array}\right]\left[\begin{array}{ll}
\operatorname{Re}\left(\boldsymbol{\Psi}_{d_{j}}\right) & \operatorname{Im}\left(\boldsymbol{\Psi}_{d_{j}}\right) \\
\operatorname{Im}\left(\bar{\Psi}_{d_{j}}\right) & \operatorname{Re}\left(\overline{\boldsymbol{\Psi}}_{d_{j}}\right)
\end{array}\right]\left[\begin{array}{c}
\widetilde{\mathbf{c}}_{j} \\
\widetilde{\mathbf{c}}_{j+1}
\end{array}\right]
\end{aligned}
$$

where the real and imaginary parts of the (in this case complex) matrix $\boldsymbol{\Psi}_{d_{j}}$ introduced in (65) are

$$
\begin{aligned}
& \operatorname{Re}\left(\boldsymbol{\Psi}_{d_{j}}\right)=\operatorname{Re}\left(\overline{\boldsymbol{\Psi}}_{d_{j}}\right)=\left[\begin{array}{ll}
e^{\lambda_{j} z} \cos \mu_{j} z & e^{-\lambda_{j} z} \cos \mu_{j} z
\end{array}\right] \\
& \operatorname{Im}\left(\boldsymbol{\Psi}_{d_{j}}\right)=-\operatorname{Im}\left(\bar{\Psi}_{d_{j}}\right)=\left[\begin{array}{ll}
e^{\lambda_{j} z} \sin \mu_{j} z & -e^{-\lambda_{j} z} \sin \mu_{j} z
\end{array}\right]
\end{aligned}
$$

whereby the real formulation in the right-hand side of equation (84) becomes

$$
\begin{aligned}
{\left[\begin{array}{ll}
\mathbf{a}_{j} & \mathbf{b}_{j}
\end{array}\right]\left[\begin{array}{rl}
e^{\lambda_{j} z} \cos \mu_{j} z & e^{-\lambda_{j} z} \cos \mu_{j} z \\
-e^{\lambda_{j} z} \sin \mu_{j} z & e^{-\lambda_{j} z} \sin \mu_{j} z \\
e^{\lambda_{j} z} \sin \mu_{j} z & -e^{-\lambda_{j} z} \sin \mu_{j} z \\
e^{\lambda_{j} z} \cos \mu_{j} z & e^{-\lambda_{j} z} \cos \mu_{j} z
\end{array}\right]\left[\begin{array}{c}
\widetilde{c}_{j_{1}} \\
\widetilde{c}_{j_{2}} \\
\widetilde{c}_{j_{1+1}} \\
\bar{c}_{j_{1+1}}
\end{array}\right] }
\end{aligned}
$$

This allows us to rewrite the complex quantities into real quantities. Let us modify the modal matrices $\mathbf{V}_{\mathrm{w}}$ and $\mathbf{V}_{\Omega}$ and introduce the modified modal matrices $\widetilde{\mathbf{V}}_{\mathrm{W}}$ and $\widetilde{\mathbf{V}}_{\Omega}$ by substituting the complex pairs of eigenvectors with their respective real and imaginary parts. Further more let us also introduce the modified solution matrix $\widetilde{\boldsymbol{\Psi}}$ and its related modified vector of constants $\widetilde{\mathbf{c}}$ by substituting the solutions (and constants) of the complex pairs using equation (84), i.e. equation (86). This allows us to write the full homogeneous solution along the beam with real numbers as

$$
\begin{aligned}
\mathbf{u}_{\mathrm{w}}(z) & =\widetilde{\mathbf{V}}_{\mathrm{w}} \widetilde{\boldsymbol{\Psi}}(z) \widetilde{\mathbf{c}} \\
\mathbf{u}_{z}(z) & =-\widetilde{\mathbf{V}}_{\Omega} \widetilde{\boldsymbol{\Psi}}^{\prime}(z) \widetilde{\mathbf{c}}
\end{aligned}
$$


We may choose to work with this real formulation or continue working with complex numbers using the full homogeneous solution formulated in equation (79). Before elaborating on how to find the solution constants, we will look at a couple of examples, showing the different modes and eigenvalues.

\section{Examples of transverse and axial displacement modes}

Using the approach described, it is possible to identify all the eigenvectors of the current GBT formulation, which is based on simple constitutive relations, discretization of the cross-section displacements in combination with a weak formulation of the shear constraints and constrained wall width. The approach allows direct analysis of both open and closed thin-walled crosssections without special considerations. Furthermore, the mathematical solution of the problem is not approximate, but performed as in non-proportionally damped modal analysis.

The following three examples illustrate the eigenmodes related to the lowest eigenvalues of the formulated eigenvalue problem for generalized thin-walled beams. The first example relates to an open channel cross-section and the second relates to a closed rectangular box cross-section. The third example illustrates the $3 \mathrm{D}$ behavior of distortional eigensolutions. The examples show that some of the important distortional modes and related eigenvalues are complex. We have chosen to show both real and imaginary parts of all the eigenmodes in order to illustrate that they come in pairs and that the imaginary part of the conjugated mode changes sign. However as we have seen in section 8.2 the conjugated pair can be replaced by two real modes corresponding to the real and the imaginary part of one of the pair bearing in mind that these two are then coupled and interact along the beam. Even though we in our examples only show results for real local modes we have to state that a few of the higher modes become complex, therefor we are not, on the basis of these examples, able to draw conclusions on this.

The examples also illustrate that the pure St. Venant twist mode is included with a linear axial solution for both closed and open sections, while the exponential solution with pure rigid cross-section twist does not seem to exist for the closed rectangular cross-section, and an investigation of the mode found for the open crosssection shows that it contains very small distortions.

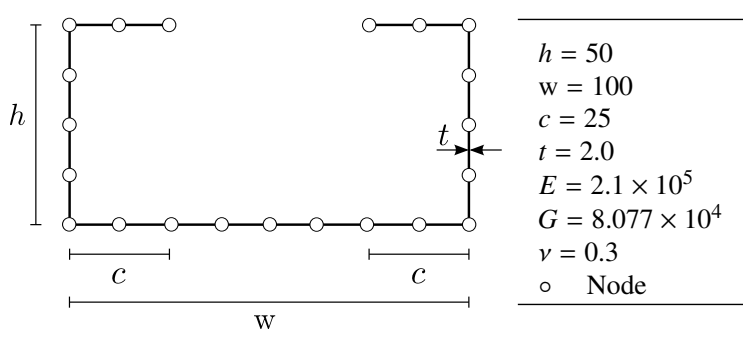

Figure 5: Geometry and parameter values of a lipped channel.

\subsection{Example 1 - Eigenmodes of a lipped channel cross-section}

Solving the generalized eigenvalue problem in equation (61) using geometric parameters and values as given in Figure 5 leads to a number of modes corresponding to the number of degrees of freedom. The first thirteen eigenvalues are shown in Table 6. For this lipped channel cross-section, modes $0,1,2,3$ are beam modes, modes $4,5,6,7,8$ are non-local distortional, and the remaining modes 9-84 are local distortional modes. The designation of local and non-local distortional modes is made on the basis of the difference in the attenuation of the modes (difference in problem length scales) seen in relation to the typical dimensions of the cross-section. Mode 4 is the exponentially varying counterpart to pure St. Venant twist, but it also contains wall distortions, which in this example are in the order of magnitude $10^{-3}$ of the twist displacements, and in this formulation it is therefore an independent mode. The in-plane mode shapes corresponding to the eigenvalues in Table 6 are shown in Figure 6, while the corresponding warping mode shapes are shown in Figure 7. Note that the imaginary part of the mode vector is about 10-100 times smaller than the real counterpart. The modes found seem very similar to the most important modes found by Silvestre in [13]. Note that Silvester does not comment on the choice of numbering order, where the modes in this paper are ordered according to the attenuation length.

\subsection{Example 2 - Eigenmodes of a rectangular box cross-section}

Using the geometric parameters and values as given in Figure 8 leads to a number of modes corresponding to the number of degrees of freedom. The first ten calculated eigenvalues are shown in Table 7 . For this channel cross-section, modes $0,1,2,3$ are beam modes, modes 4, 5 are non-local distortional modes, and the remaining modes 6-94 are local distortional modes. Note that there is no exponentially varying counterpart to pure St. 


\begin{tabular}{|c|c|c|c|c|c|}
\hline \multicolumn{3}{|c|}{ Mode } & \multicolumn{2}{|c|}{ Eigenvalues } & \multirow{2}{*}{$\begin{array}{c}\text { Axial solution } \\
\psi(z)\end{array}$} \\
\hline & ype & No. & $\xi^{2} \times 10^{6}$ & $\xi \times 10^{3}$ & \\
\hline صี & $\begin{array}{l}\overline{0} \\
\frac{0}{00}\end{array}$ & $\begin{array}{l}0 \\
1 \\
2 \\
3\end{array}$ & $\begin{array}{l}0 \\
0 \\
0 \\
0\end{array}$ & $\begin{array}{l}0 \\
0 \\
0 \\
0\end{array}$ & $\begin{array}{c}-c_{a 1} z-c_{a 2} z^{2} \\
c_{11}+c_{12} z+c_{13} z^{2}+c_{14} z^{3} \\
c_{21}+c_{22} z+c_{23} z^{2}+c_{24} z^{3} \\
c_{31}+c_{32} z\end{array}$ \\
\hline \multirow{2}{*}{ : } & 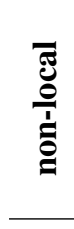 & $\begin{array}{l}4 \\
5 \\
6 \\
7 \\
8 \\
\end{array}$ & $\begin{array}{c}0.37 \\
3.36-26.52 i \\
3.36+26.52 i \\
4.23-50.04 i \\
4.23-50.04 i\end{array}$ & $\begin{array}{c}0.61 \\
\pm(3.88-3.42 i) \\
\pm(3.88+3.42 i) \\
\pm(5.22-4.80 i) \\
\pm(5.22+4.80 i)\end{array}$ & $\begin{array}{c}c_{d 1} e^{\xi z}+c_{d 2} e^{-\xi z} \\
c_{d 3} e^{\xi z}+c_{d 4} e^{-\xi z} \\
c_{d} e^{\xi z}+c_{d 6} e^{-\xi z} \\
c_{d 7} e^{\xi z}+c_{d 8} e^{-\xi z} \\
c_{d 9} e^{\xi z}+c_{d 10} e^{-\xi z}\end{array}$ \\
\hline & ฮ్ & $\begin{array}{c}9 \\
10 \\
11 \\
12\end{array}$ & $\begin{array}{l}825.6 \\
951.1 \\
1823 \\
3359\end{array}$ & $\begin{array}{l}28.7 \\
30.8 \\
42.7 \\
58.0\end{array}$ & $\begin{array}{l}c_{d 11} e^{\xi z}+c_{d 12} e^{-\xi z} \\
c_{d 13} e^{\xi z}+c_{d 14} e^{-\xi z} \\
c_{d 15} e^{\xi z}+c_{d 16} e^{-\xi z} \\
c_{d 17} e^{\xi z}+c_{d 18} e^{-\xi z}\end{array}$ \\
\hline
\end{tabular}

Table 6: Eigenvalues $\xi^{2}$, the $\xi$ value and the related axial solutions for the lipped channel cross-section.

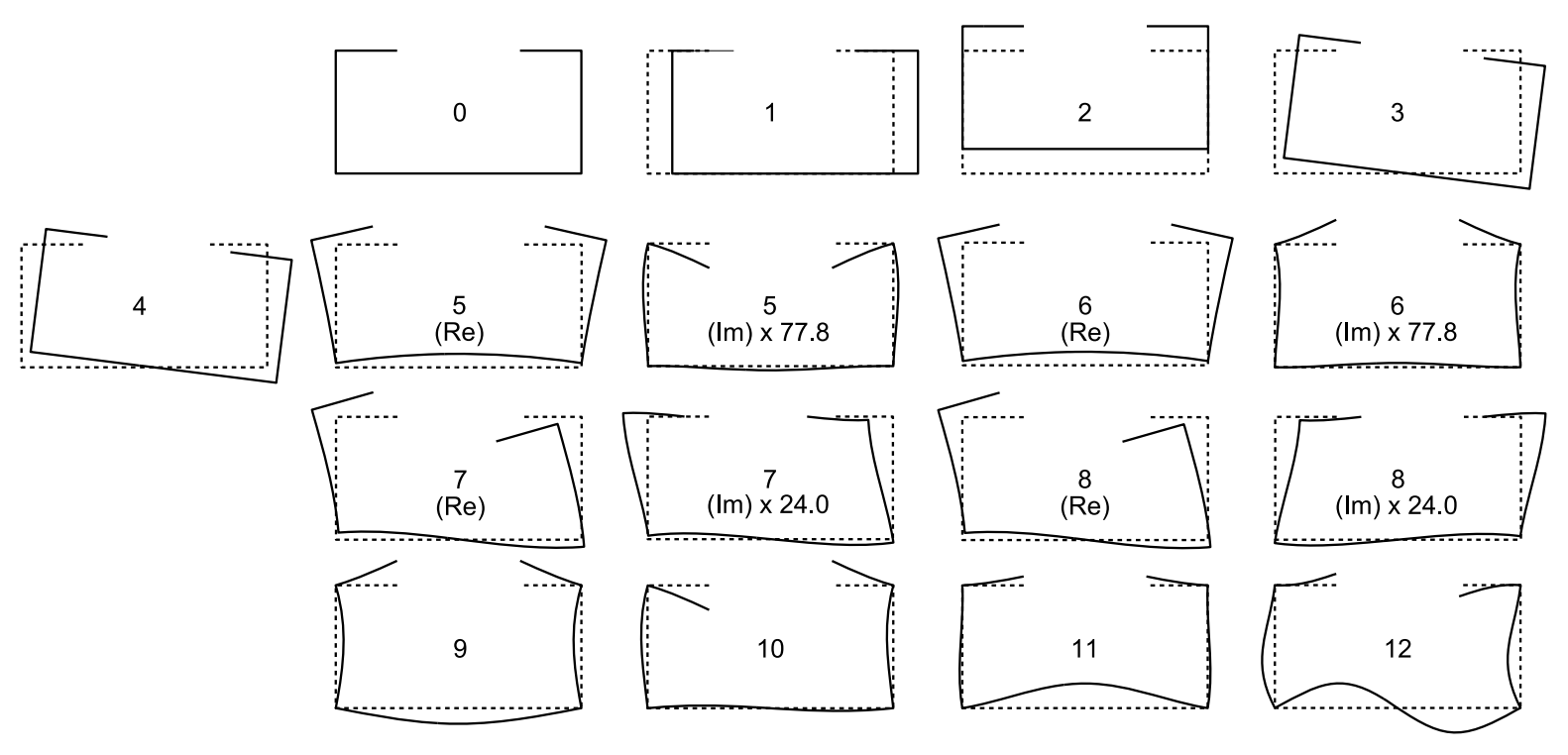

Figure 6: Lipped channel - 13 in-plane deformation mode shapes. 


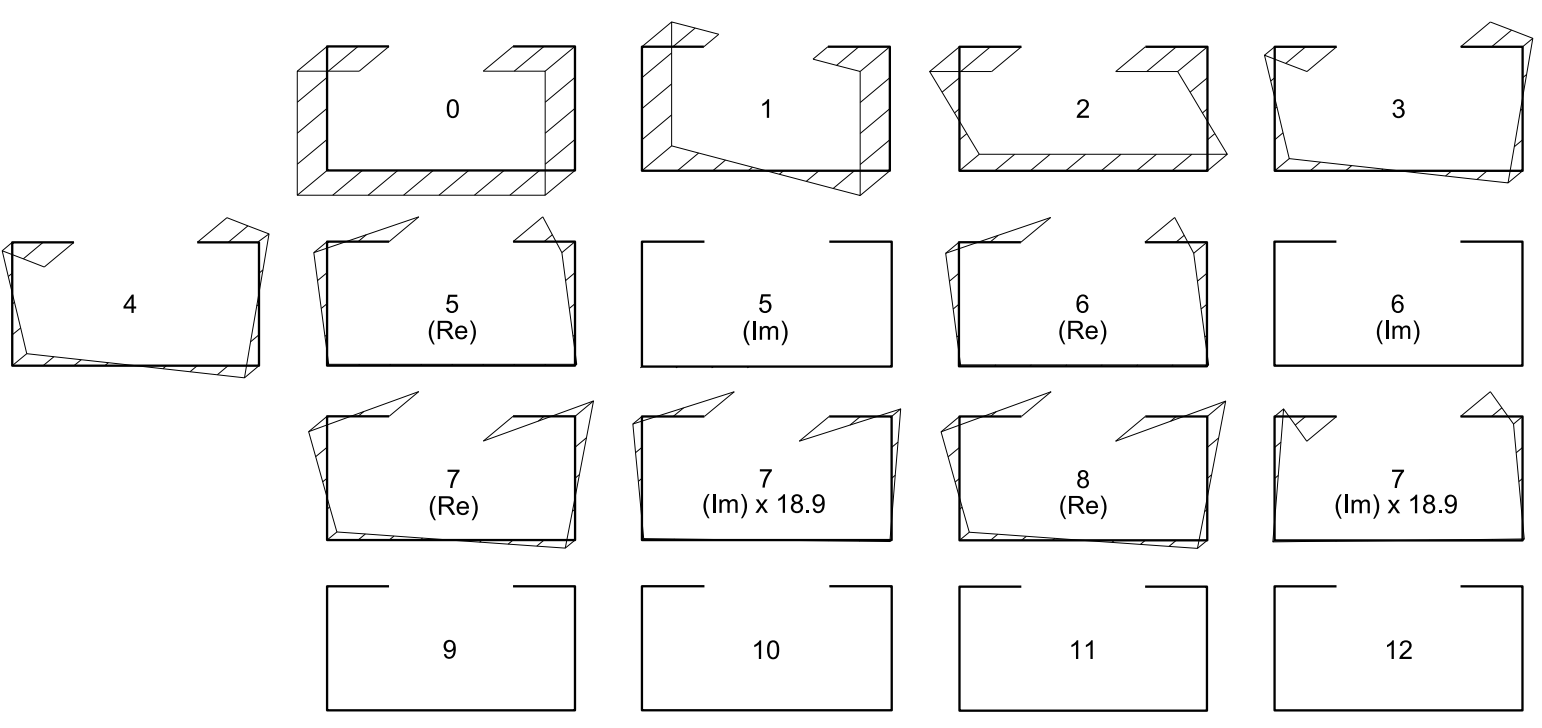

Figure 7: Lipped channel - 13 warping deformation mode shapes.

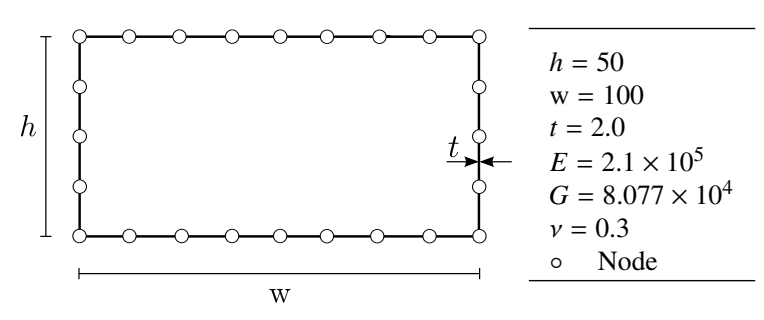

Figure 8: Geometry and parameter values of a rectangular section.

Venant twist. The in-plane mode shapes corresponding to the eigenvalues in Table 7 are shown in Figure 9, while the corresponding warping mode shapes are shown in Figure 10. Also note that for the closed section the imaginary part of the mode vector is $10-100$ times smaller than its real counterpart. The modes found seem very similar to the modes found by Gonçalves \& Camotim in [4] and seem to span the same deformation space.

\subsection{Example 3 - Two distortional eigensolutions for both channel and box beams}

In this example we show the two eigensolutions related to the first complex distortional displacement mode of the channel and box cross-sections. We choose to illustrate the mode corresponding to the first complex distortional displacement mode, which are mode $j=5$ and $j=4$ respectively for the channel and box cross-sections. With the use of equation (86) in which the real part, a, and the imaginary part, $\mathbf{b}$, of the mode are multiplied by the solution functions. We choose in Figure 11 to illustrate two of the four independent solution functions: one in which only $\widetilde{c}_{j_{2}}$ is non- zero (shown as a. and b. in the figure), and the other (shown as c. and d.) in which only $\widetilde{c}_{j+1}$ is non zero. Thus sub figure $a$. and $b$. correspond to the solution $e^{-\lambda_{j} z} \cos \mu_{j} z \cdot \mathbf{a}_{j}+e^{-\lambda_{j} z} \sin \mu_{j} z \cdot \mathbf{b}_{j}$ and sub figure c. and d. corresponds to $-e^{-\lambda_{j} z} \sin \mu_{j} z \cdot \mathbf{a}_{j}+e^{-\lambda_{j} z} \cos \mu_{j} z \cdot \mathbf{b}_{j}$. The eigensolutions shown in the figure therefore involves a coupled behavior of the real part and imaginary part of the mode.

\section{The degree of freedom space and related trans- formations}

To apply the present work and make use of the solutions found in a finite element context, it is necessary to be able to relate to the different degree-of-freedom spaces in use as well as to the constraints introduced. In Step I, the introduction of shear constraints leads to a generalized beam theory (GBT) in which only shear flow around closed cells is taken into account while all other shears are constrained. With the exception of pure axial extension, the axial displacements are determined from the axial derivative of the transverse displacements. From the boundary terms of the first variation of the potential energy given in equation (27), it is seen that the (virtual) generalized boundary displacements are pure axial extension $\left(\zeta v_{\Omega}^{a}\right)$, transverse displacements $\left(\psi \mathbf{v}_{\mathrm{w}}\right)$, and the axial derivative of the transverse displacements $\left(\psi \mathbf{v}_{\mathrm{w}}\right)^{\prime}$. However, the transverse displacements are unconstrained, which is not compatible with classical Vlasov beam theory where the individual thin wall of the cross-section is assumed to 


\begin{tabular}{|c|c|c|c|c|c|}
\hline \multicolumn{3}{|c|}{ Mode } & \multicolumn{2}{|c|}{ Eigenvalues } & \multirow{2}{*}{$\begin{array}{c}\text { Axial solution } \\
\psi(z) \\
\end{array}$} \\
\hline & pe & No. & $\xi^{2} \times 10^{6}$ & $\xi \times 10^{3}$ & \\
\hline 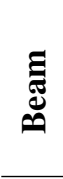 & $\frac{\bar{\pi}}{\frac{0}{0}}$ & $\begin{array}{l}0 \\
1 \\
2 \\
3\end{array}$ & $\begin{array}{l}0 \\
0 \\
0 \\
0\end{array}$ & $\begin{array}{l}0 \\
0 \\
0 \\
0\end{array}$ & $\begin{array}{c}-c_{a 1} z-c_{a 2} z^{2} \\
c_{11}+c_{12} z+c_{13} z^{2}+c_{14} z^{3} \\
c_{21}+c_{22} z+c_{23} z^{2}+c_{24} z^{3} \\
c_{31}+c_{32} z\end{array}$ \\
\hline 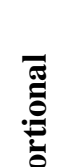 & 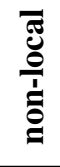 & $\begin{array}{l}4 \\
5\end{array}$ & $\begin{array}{l}0.72-36.95 i \\
0.72+36.95 i\end{array}$ & $\begin{array}{l} \pm(4.34-4.26 i) \\
\pm(4.34+4.26 i)\end{array}$ & $\begin{array}{l}c_{d 1} e^{\xi z}+c_{d 2} e^{-\xi z} \\
c_{d 3} e^{\xi z}+c_{d 4} e^{-\xi z}\end{array}$ \\
\hline$\ddot{\ddot{n}}$ & త్ర & $\begin{array}{l}6 \\
7 \\
8 \\
9\end{array}$ & $\begin{array}{l}1205 \\
2661 \\
2050 \\
4837 \\
\end{array}$ & $\begin{array}{l}34.7 \\
51.6 \\
45.3 \\
69.6 \\
\end{array}$ & $\begin{array}{c}c_{d 5} e^{\xi z}+c_{d 6} e^{-\xi z} \\
c_{d 7} e^{\xi z}+c_{d 8} e^{-\xi z} \\
c_{d 9} e^{\xi z}+c_{d 10} e^{-\xi z} \\
c_{d 11} e^{\xi z}+c_{d 12} e^{-\xi z}\end{array}$ \\
\hline
\end{tabular}

Table 7: Eigenvalues $\xi^{2}$, the $\xi$ value and the related axial solutions for the rectangular box cross-section.
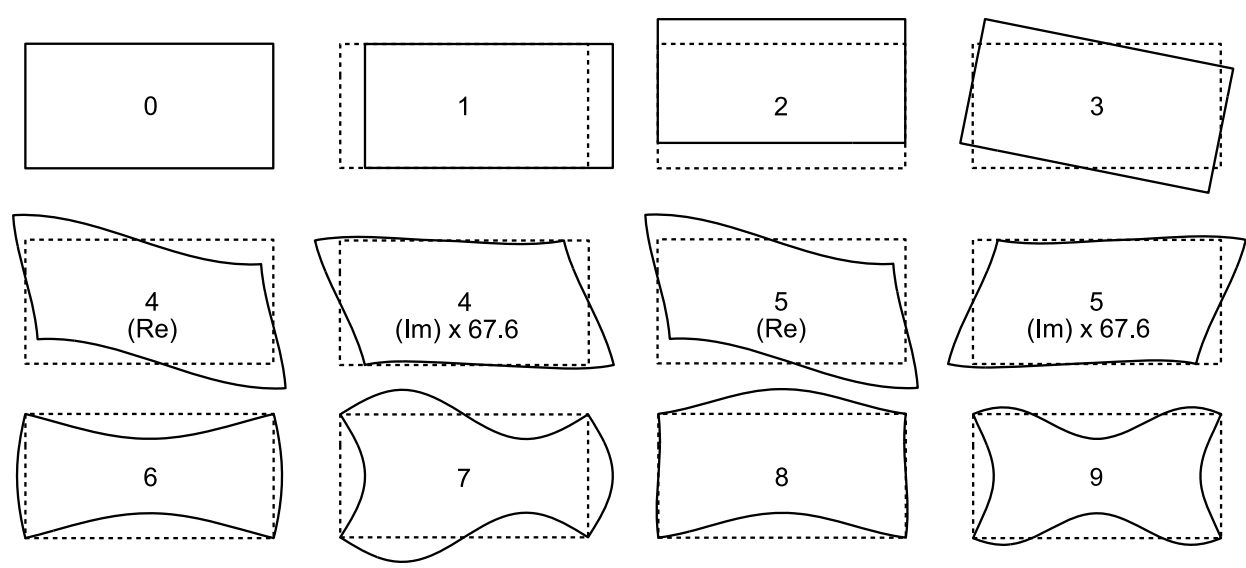

Figure 9: Rectangular box cross-section - 10 in-plane deformation mode shapes.
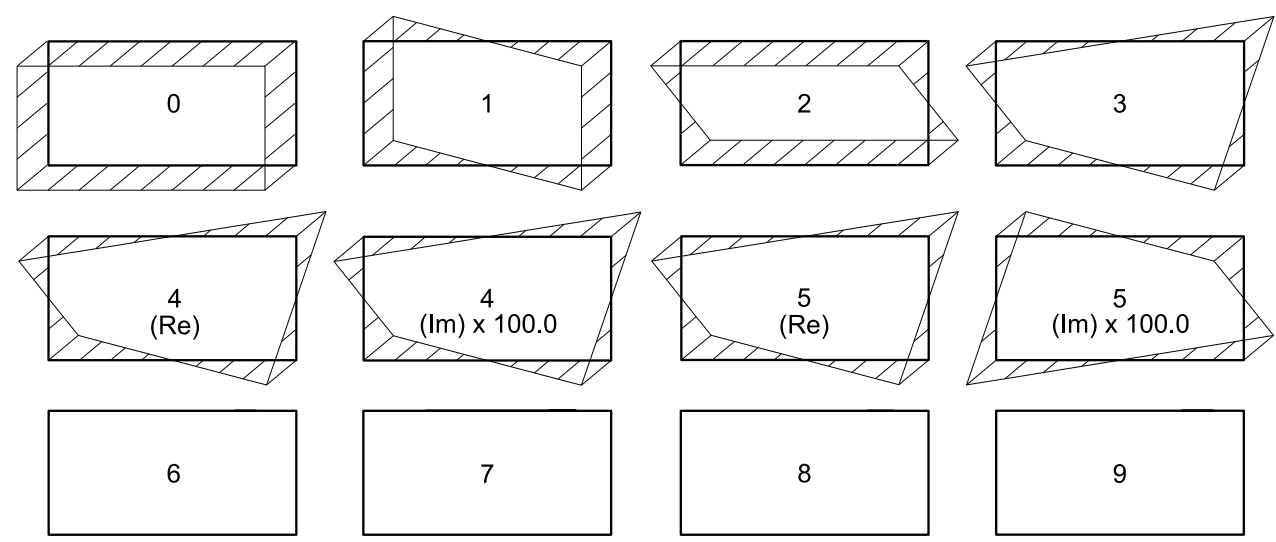

Figure 10: Rectangular box cross-section - 10 warping deformation mode shapes. 


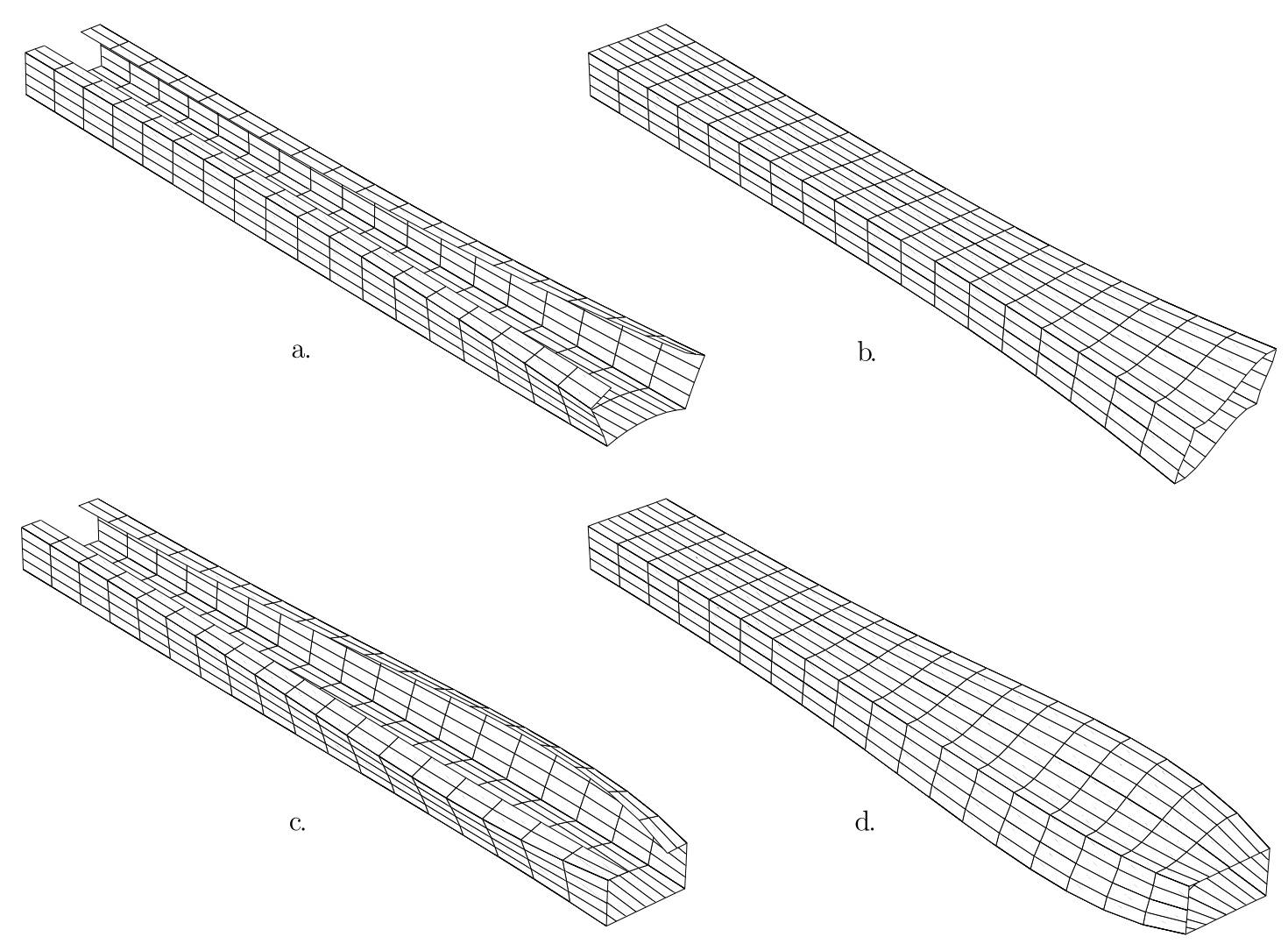

Figure 11: Two eigensolutions of the first complex distortional mode for a channel and a box section.

maintain its length (width) within the cross-section, i.e. no cross-section centerline elongation. This is overcome in Step II where the walls are constrained using a set of multipoint constraint equations which eliminate constrained transverse displacement degrees of freedom $\mathbf{v}_{\mathrm{w}}^{c}$. The basic degrees of freedom of the GBT formulation are the pure axial extension $\left(\zeta v_{\Omega}^{a}\right)$, the remaining transverse displacements $\left(\psi \mathbf{v}_{\mathrm{w}}^{g}\right)$, and the axial derivative hereof $\left(\psi \mathbf{v}_{\mathrm{W}}^{g}\right)^{\prime}$. To be able to change degree-of-freedom space from GBT space $\mathbf{v}_{\mathrm{W}}^{g}$ to finite element (FE) (original degree-of-freedom) space $\mathbf{v}_{\mathrm{w}}$, the following transformation is introduced:

$\mathbf{v}_{\mathrm{w}}=\left[\begin{array}{ll}\mathbf{T}_{\mathrm{w}}^{c} & \mathbf{T}_{\mathrm{w}}^{g}\end{array}\right]\left[\begin{array}{c}\mathbf{v}_{\mathrm{W}}^{c} \\ \mathbf{v}_{\mathrm{w}}^{g}\end{array}\right]$

where $\mathbf{T}_{\mathrm{w}}^{g}=\left[\begin{array}{ll}\mathbf{T}_{\mathrm{w}}^{i} & \mathbf{T}_{\mathrm{w}}^{u}\end{array}\right]$ and $\mathbf{T}_{\mathrm{w}}^{i}=\left[\begin{array}{ccc}1 & 0 & 0 \\ 0 & 1 & 0 \\ \vdots & 0 & 1 \\ & \vdots & 0 \\ & & \vdots\end{array}\right]$

in which $\mathbf{T}_{\mathrm{w}}^{c}$ and $\mathbf{T}_{\mathrm{w}}^{u}$ have already been introduced in Step II, and $\mathbf{T}_{\mathrm{w}}^{i}$ is a matrix corresponding to three sup- plementary columns, which pick out the degrees of freedom of the first node related to $v_{\mathrm{w}}^{1}, v_{\mathrm{w}}^{2}$ and $v_{\mathrm{w}}^{3}$.

\subsection{From FE displacements to GBT displacements}

If the transverse displacement vector $\mathbf{v}_{\mathrm{w}}$ already fulfills the constraint equations, then we can find the GBT transverse displacement vector by using $\mathbf{T}_{\mathrm{w}}^{g T}$, and the pure axial extension by using $\mathbf{T}_{\Omega}^{a T}$ as follows:

$$
\left[\begin{array}{c}
\mathbf{v}_{\mathrm{W}}^{g} \\
v_{\Omega}^{a}
\end{array}\right]=\left[\begin{array}{cc}
\mathbf{T}_{\mathrm{W}}^{g T} & \mathbf{0} \\
\mathbf{0} & \mathbf{T}_{\Omega}^{a T}
\end{array}\right]\left[\begin{array}{l}
\mathbf{v}_{\mathrm{W}} \\
\mathbf{v}_{\Omega}
\end{array}\right]
$$

This is the important transformation from FE space to GBT space, which we will need to be able to find the constants of the homogeneous solution.

\subsection{From GBT displacements to FE displacements}

However, we may at some point also need the opposite transformation, which involves the constraint equations introduced in equation (42). Let us principally use the same method but introduce the transformation equation (88) whereby the multi-point constraint equations take the form

$$
\mathbf{C} \mathbf{T}_{\mathrm{w}}^{c} \mathbf{v}_{\mathrm{w}}^{c}+\mathbf{C} \mathbf{T}_{\mathrm{w}}^{g} \mathbf{v}_{\mathrm{w}}^{g}=\mathbf{0} \quad \Leftrightarrow \quad \mathbf{C}_{c} \mathbf{v}_{\mathrm{w}}^{c}+\mathbf{C}_{g} \mathbf{v}_{\mathrm{w}}^{g}=\mathbf{0}
$$


in which $\mathbf{C}_{c}=\mathbf{C} \mathbf{T}_{\mathrm{w}}^{c}$ has previously been introduced and $\mathbf{C}_{g}=\mathbf{C} \mathbf{T}_{\mathrm{W}}^{g}$ is introduced here. This allows us to express the constrained degrees of freedom by the GBT transverse displacement vector as:

$\mathbf{v}_{\mathrm{w}}^{c}=-\mathbf{C}_{c}^{-1} \mathbf{C}_{g} \mathbf{v}_{\mathrm{W}}^{g}$

Introducing the equality (91) in the transformation equation (88), we find that the total transformation condenses our problem as follows:

$\mathbf{v}_{\mathrm{w}}=\left[\begin{array}{ll}\mathbf{T}_{\mathrm{w}}^{c} & \mathbf{T}_{\mathrm{w}}^{g}\end{array}\right]\left[\begin{array}{c}-\mathbf{C}_{c}^{-1} \mathbf{C}_{g} \mathbf{v}_{\mathrm{w}}^{g} \\ \mathbf{v}_{\mathrm{w}}^{g}\end{array}\right]=\widetilde{\mathbf{T}}_{\mathrm{w}}^{g} \mathbf{v}_{\mathrm{w}}^{g}$

where $\widetilde{\mathbf{T}}_{\mathrm{w}}^{g}=\mathbf{T}_{\mathrm{w}}^{g}-\mathbf{T}_{\mathrm{w}}^{c} \mathbf{C}_{c}{ }^{-1} \mathbf{C}_{g}$ has been introduced as the condensed transformation. Using the transformation equation (24) that determines the warping displacements from the amount of axial extension and the transverse displacements we find

$$
\mathbf{v}_{\Omega}=\left[\begin{array}{ll}
\mathbf{T}_{\Omega \mathrm{w}}^{r} & \mathbf{T}_{\Omega}^{a}
\end{array}\right]\left[\begin{array}{c}
\mathbf{v}_{\mathrm{w}} \\
v_{\Omega}^{a}
\end{array}\right]=\left[\begin{array}{lll}
\mathbf{T}_{\Omega \mathrm{W}}^{r} & \widetilde{\mathbf{T}}_{\mathrm{W}}^{g} & \mathbf{T}_{\Omega}^{a}
\end{array}\right]\left[\begin{array}{c}
\mathbf{v}_{\mathrm{W}}^{g} \\
v_{\Omega}^{a}
\end{array}\right]
$$

Using equations (92) and (93) we find the following transformation

$\left[\begin{array}{c}\mathbf{v}_{\mathrm{W}} \\ \mathbf{v}_{\Omega}\end{array}\right]=\left[\begin{array}{cc}\widetilde{\mathbf{T}}_{\mathrm{w}}^{g} & \mathbf{0} \\ \mathbf{T}_{\Omega \mathrm{w}}^{r} \widetilde{\mathbf{T}}_{\mathrm{W}}^{g} & \mathbf{T}_{\Omega}^{a}\end{array}\right]\left[\begin{array}{c}\mathbf{v}_{\mathrm{W}}^{g} \\ v_{\Omega}^{a}\end{array}\right]$

This transformation is used to transform from GBT space to FE space.

\section{Displacement boundary conditions of the homo- geneous solution}

Having solved the eigenvalue problem and formulated solution modes in the original FE degree-offreedom space, we would like to set up a method for determining the constants of the homogeneous solutions found. This is to be done in the GBT space. As seen from the first variation of the potential energy, the natural boundary displacements of the GBT at each boundary are the pure axial displacement $u_{\Omega}^{a}$ of the beam, the transverse displacements $\mathbf{u}_{\mathrm{w}}^{g}$, and the axial derivative of the transverse displacements $\mathbf{u}_{\mathrm{w}}^{g}$. . The generalized internal displacements of the GBT beam can be expressed by using the full homogeneous solution in equation (79) or alternatively, as done in the following, by the real formulation in equation (87) and the transformation from FE to GBT displacements (89) as follows:

$$
\left[\begin{array}{c}
u_{z}^{a}(z) \\
\mathbf{u}_{\mathrm{w}}^{g}(z) \\
\mathbf{u}_{\mathrm{w}}^{g \prime}(z)
\end{array}\right]=\left[\begin{array}{c}
-\mathbf{T}_{\Omega}^{a T} \widetilde{\mathbf{V}}_{\Omega} \widetilde{\boldsymbol{\Psi}}^{\prime}(z) \\
\mathbf{T}_{\mathrm{w}}^{g T} \widetilde{\mathbf{V}}_{\mathrm{w}} \widetilde{\boldsymbol{\Psi}}(z) \\
\mathbf{T}_{\mathrm{w}}^{g} T \widetilde{\mathbf{V}}_{\mathrm{w}} \widetilde{\boldsymbol{\Psi}}^{\prime}(z)
\end{array}\right] \widetilde{\mathbf{c}}
$$

To determine the constants using displacement boundary conditions as in finite element or stiffness formulations, we need the boundary displacements at the two ends of a finite length beam, i.e. at $z=0$ and at $z=L$ where $L$ is the length of the beam. The assembled boundary displacement vector is denoted by $\mathbf{u}_{b}$ This leads to the following equation for the determination of the solution constants:

$$
\begin{aligned}
& \mathbf{u}_{b}=\left[\begin{array}{c}
u_{z}^{a}(0) \\
\mathbf{u}_{\mathrm{w}}^{g}(0) \\
\mathbf{u}_{\mathrm{w}}^{g \prime}(0) \\
u_{z}^{a}(L) \\
\mathbf{u}_{\mathrm{w}}^{g}(L) \\
\mathbf{u}_{\mathrm{w}}^{g \prime}(L)
\end{array}\right]=\left[\begin{array}{r}
-\mathbf{T}_{\Omega}^{a T} \widetilde{\mathbf{V}}_{\Omega} \widetilde{\boldsymbol{\Psi}}^{\prime}(0) \\
\mathbf{T}_{\mathrm{w}}^{g} T \widetilde{\mathbf{V}}_{\mathrm{w}} \widetilde{\boldsymbol{\Psi}}(0) \\
\mathbf{T}_{\mathrm{w}}^{g T} \widetilde{\mathbf{V}}_{\mathrm{w}} \widetilde{\mathbf{\Psi}}^{\prime}(0) \\
-\mathbf{T}_{\Omega}^{a T} \widetilde{\mathbf{V}}_{\Omega} \widetilde{\mathbf{\Psi}}^{\prime}(L) \\
\mathbf{T}_{\mathrm{w}}^{g} T \widetilde{\mathbf{V}}_{\mathrm{w}} \widetilde{\Psi^{\prime}}(L) \\
\mathbf{T}_{\mathrm{w}}^{g T} \widetilde{\mathbf{V}}_{\mathrm{w}} \widetilde{\Psi}^{\prime}(L)
\end{array}\right] \widetilde{\mathbf{c}}=\widetilde{\mathbf{A}} \widetilde{\mathbf{c}} \\
& \Rightarrow \widetilde{\mathbf{c}}=\widetilde{\mathbf{A}}^{-1} \mathbf{u}_{b}
\end{aligned}
$$

where we have introduced the matrix $\widetilde{\mathbf{A}}$, which is an invertible positive definite "square" matrix. However, to avoid numerical problems, the exponential solution functions in $\widetilde{\boldsymbol{\Psi}}(z)$ may have to be modified by replacing $\widetilde{c}_{i} e^{\lambda z}$ by $\hat{c}_{i} e^{\lambda(z-L)}$ so that the positive $\lambda z$ exponent is bounded.

The two solutions plotted by using equation (87) with only $\widetilde{c}_{j_{2}}$ being non zero in the upper half of Figure 11 can also be found by using the relevant boundary conditions in equation (97). To this end, we use the real and imaginary vectorial parts, $\mathbf{a}, \mathbf{b}$, of the mode shape and the modal solution functions and the derivative hereof. So in equation (97), we would use

$\mathbf{u}_{b}=\left[\begin{array}{c}0 \\ \mathbf{a} \\ -\lambda \mathbf{a}-\mu \mathbf{b} \\ 0 \\ e^{-\lambda L}(\mathbf{a} \cos \mu L+\mathbf{b} \sin \mu L) \\ -e^{-\lambda L}\{\mathbf{a}(\lambda \cos \mu L+\mu \sin \mu L)+\mathbf{b}(\lambda \sin \mu L-\mu \cos \mu L)\}\end{array}\right]$

and we should then find only the second constant of the $j$ 'th complex mode $\widetilde{c}_{j_{2}}$ to be non zero. The two lower solutions plotted in Figure 11 correspond to boundary conditions in which only the fourth constant $\widetilde{c}_{j_{4}}$ of the complex mode is non-zero.

It is also worth noting that just specifying the modal shape with a zero derivative (otherwise built-in support) will lead to a coupling to the remaining modes. For example, Figure 12 shows the displacements for the boundary displacements corresponding to the real part of the first complex distortional eigenmode at one end, but with zero axial displacements and zero displacements and axial displacement derivatives at the other end, i.e. with the following boundary condition: 

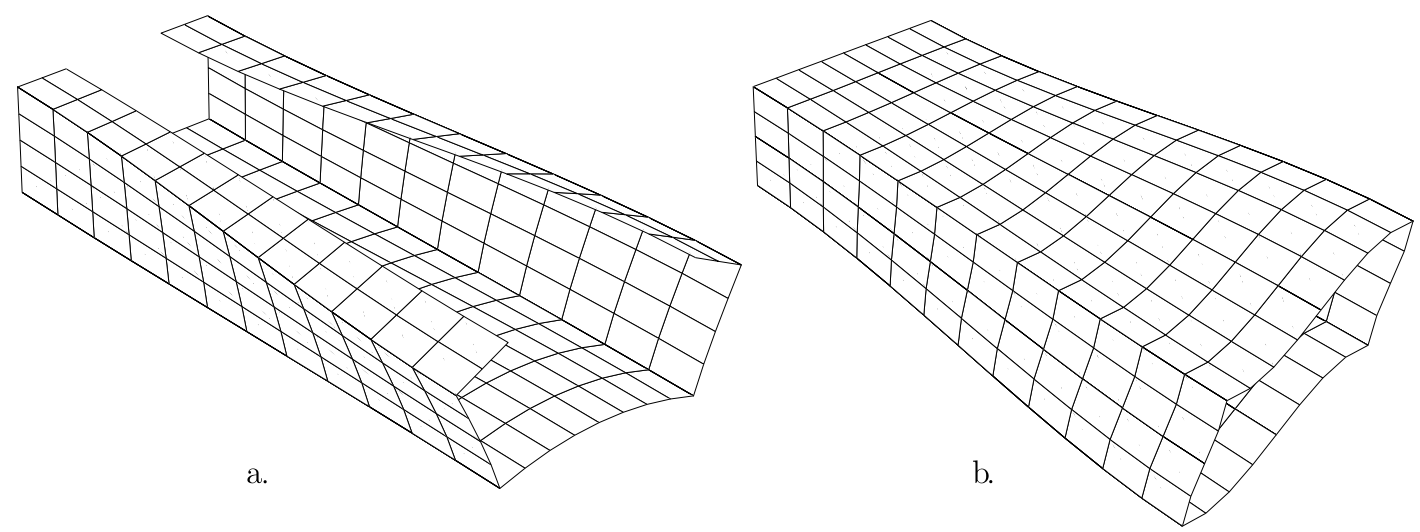

Figure 12: Displacement field for boundary conditions in equation (99).

With this boundary condition many modes are invoked and to achieve the zero derivative of the displacements, local distortional plate modes have also been invoked. The problem length scales of some of these modes are very small and will be difficult to see in an overall plot of the deformation mode. The formulation above enables finite element formulation of advanced semi-discretized thin-walled beam elements.

\section{Conclusion}

We have presented a new systematic method accompanied by a detailed description for the whole semidiscretization process from kinematic assumptions, potential energy, potential energy variation leading to the formulation of the homogeneous differential equations of a generalized beam theory (GBT) and the establishment of the full solution through identification of all eigenvalues and eigenmodes. This new approach is a considerable theoretical and practical development, since the obtained GBT equations are now solved analytically and the formulation is valid without special attention also for closed single or multi cell crosssections.

The beam displacement field was separated into a sum of products of the cross-section displacement modes and their axial variation. This displacement field was constrained to follow the shear assumptions made in Vlasov beam theory by a weak formulation of the constant shear flow assumption. This allows the identical treatment of both open and closed cross-sections.

The coupled homogeneous fourth order differential equations of GBT have not been solved approximately, but by a reduction of order accompanied by a doubling of the number of equations and the introduction of a state vector, as in non-proportionally damped dynamic analysis. In traditional GBT analysis, the eigenvalue problem, which defines the distortional modes corresponds to the solution of the undamped eigenvalue problem solved in proportionally damped dynamic structural analysis, (i.e. by neglecting the shear stiffness matrix related to torsion). However the related eigenvalues have not been used for assessment of the attenuation behavior.

It is clear that the eigenvalues of the distortional modes found are inverse length scale parameters or attenuation parameters which define the axial solution functions and allow us to predict the length of the distortional displacement field. This may be used to determine the degree of discretization if the eigenmodes found are to be used in a traditional type GBT finite element formulation with a reduced number of modes and with approximate shape functions for the axial variation. Alternatively future formulation of distortional beam elements may be based on the found distortional modes with axial variations based on the analytical solution functions. In a following paper we will address the solution of the inhomogeneous GBT equations using the eigenmodes found in this paper in order to decouple the non-homogeneous differential equations.

The modes found in this paper seem to be similar to the modes found in traditional GBT, see e.g. [13], as well as to the modes found by the modified GBT formulation for closed cross-sections, see [4]. In this relation it will be of interest to perform a proper comparison of the found distortional modes and the modes found by the conventional GBT formulation.

\section{References}

[1] V.Z. Vlasov, Thin-walled elastic beams, 2nd ed. Israel Program for Scientific Translations, Jerusalem, Israel, 1961. 
[2] R. Schardt, Eine Erweiterung der technischen Biegelehre für die Berechnung biegesteifer prismatischer Faltwerke. Der Stahlbau, 35, 161-171, 1966.

[3] R. Schardt, Verallgemeinerte Technische Biegetheorie. SpringerVerlag, Germany, 1989.

[4] R. Gonçalves and D. Camotim, GBT local and global buckling analysis of aluminium and stainless steel columns. Computers and Structures, 82, 1473-1484, 2004.

[5] R. Gonçalves, P.B. Dinis and D. Camotim, GBT formulation to analyse the first-order and buckling behaviour of thin-walled members with arbitrary cross-sections. Thin-Walled Structures, 47, 583-600, 2009.

[6] R. Gonçalves, M. Ritto-Corrêa and D. Camotim, A new approach to the calculation of cross-section deformation modes in the framework of generalized beam theory. Computational Mechanics, 46, 759-781, 2010.

[7] C.F. Kollbrunner and N. Hajdin, Dünnwandige Stäbe 1, Stäbe mit undeformierbaren Querschnitten. Springer-Verlag, Berlin, $1972,1975$.

[8] J. Jönsson, Determination of shear stresses, warping functions and section properties of thin-walled beams using finite elements. Computers $\mathcal{E}$ Structures, 68, 393-410, 1998.

[9] J. Jönsson, Distortional warping functions and shear distributions in thin-walled beams. Thin-Walled Structures, 33, 245268, 1999.

[10] M. Hanf, Die geschlossene Lösung der linearen Differentialgleichungssysteme der Verallgemeinerten Technischen Biegetheorie mit einer Anwendung auf die Ermittlung plastischer Grenzlasten. Instituts für Werkstoffe und Mechanik im Bauwesen der TU Darmstadt, 9, 1989.

[11] J.M. Davies and P. Leach, First-order generalised beam theory. J. Construct. Steel Research, 31(2-3), 187-220, 1994.

[12] J. Jönsson, Distortional theory of thin-walled beams. ThinWalled Structures, 33, 269-303, 1999.

[13] N. Silvestre and D. Camotim, First-order generalised beam theory for arbitrary orthotropic materials. Thin-Walled Structures, 40, 755-789, 2002.

[14] N. Silvestre and D. Camotim, Second-order generalised beam theory for arbitrary orthotropic materials. Thin-Walled Structures, 40, 791-820, 2002.

[15] S. Rendek and I. Baláž, Distortion of thin-walled beams. ThinWalled Structures, 42, 255-277, 2004.

[16] N. Silvestre, Generalized Beam Theory: New formulations, Numerical Implementation and Applications. Ph.D. Thesis, IST Technical University of Lisbon, Portugal, 2005. (in Portuguese).

[17] D. Camotim, N. Silvestre, R. Gonçalves and P.B. Dinis, GBT analysis of thin-walled members: new formulations and applications. J. Loughlan eds. Thin-Walled Structures: Recent Advances and Future Trends in Thin-Walled Structures Technology, Canopus Publishing Ltd., Bath, 137-168, 2004.

[18] D. Camotim, N. Silvestre, R. Gonçalves and P.B. Dinis, GBTBased Structural Analysis of Thin-Walled Members: Overview, Recent Progress and Future Developments. M. Pandey, W.-C. $\mathrm{Xie}$ and L. Xu eds. Advances in Engineering Structures, Mechanics $\mathcal{E}$ Construction, Springer (Dordrecht), Waterloo, 187204, 2006.

[19] C.F. Kollbrunner and N. Hajdin, Dünnwandige Stäbe 2, Stäbe mit deformierbaren Querschnitten, Nicht-elastisches Verhalten dünnwandiger Stäbe. Springer-Verlag, Berlin, 1972, 1975.

[20] O.C. Zienkiewicz and R.L. Taylor, Finite Element Method 5th ed. Volume 2 - Solid Mechanics. (pp: 308). Elsevier (2000).

[21] S. Ádány and B.W. Schafer, Buckling mode decomposition of single-branched open cross-section members via finite strip method: Derivation. Thin-Walled Structures, 44, 563-584, 2006.
[22] S. Ádány and B.W. Schafer, Buckling mode decomposition of single-branched open cross-section members via finite strip method: Application and examples. Thin-Walled Structures, 44, 585-600, 2006

[23] S. Ádány and B.W. Schafer, A full modal decomposition of thin-walled, signle-branched open cross-section members via the constrained finite strip method. Journal of Constructional Steel Research, 64, 12-29, 2008.

[24] S. Ádány, N. Silvestre, B.W. Schafer and D. Camotim, GBT and cFSM: Two modal approaches to the buckling analysis of unbranched thin-walled members. International Journal of Advanced Steel Construction, 5, 195-223, 2009.

[25] S.P. Timoshenko and J.N. Goodier, Theory of Elasticity, 3rd International ed. (pp. 61). McGraw Hill Book Company, Singapore, 1970.

[26] R.D. Cook et al., Concepts and applications of finite element analysis, 4th ed. (pp. 489). John Wiley \& Sons, Inc., United States, 2001 